\title{
有机硒参与的硒环化反应研究进展
}

$\begin{array}{cccccc}\text { 许颖 } & \text { 李 晨 } & \text { 孟建萍 } & \text { 黄玉玲 } & \text { 付纪源 } \\ \text { 刘 水 } & \text { 刘颖杰 } & \text { 陈 宁* } & \\ & & \text { (哈尔滨商业大学药学院 } & \text { 哈尔滨 150076) }\end{array}$

\begin{abstract}
摘要 有机硒化物是一类重要的分子, 在药物、农用化学品、有机材料以及催化等领域有着广泛的应用, 在有机分子 中引入硒原子在合成化学中具有重要意义. 杂环化合物是构成多种生物活性分子的重要骨架, 因此, 合成含硒杂环衍 生物的研究备受关注. 分别描述了近年来快速发展的金属催化、电化学驱动、可见光驱动、有机分子催化以及其它类 型的硒环化反应, 并对部分反应的适用范围和机理进行了讨论.
\end{abstract}

关键词 有机硒化物; 杂环化合物; 硒环化; 催化

\section{Recent Progress in the Selenocyclization Reactions with Organic Selenides}

\author{
Xu, Ying Li, Chen Meng, Jianping Huang, Yuling \\ Fu, Jiyuan Liu, Bing Liu, Yingjie Chen, Ning* \\ (School of Pharmacy, Harbin University of Commerce, Harbin 150076)
}

\begin{abstract}
Organic selenides are a kind of important molecules, which are widely used in medicine, agricultural chemicals, organic materials and catalysis, the introduction of selenium atom into organic molecules is of great significance in the synthetic chemistry. Heterocyclic compounds are key skeletons involved in a variety of bioactive molecules, therefore, the development of new methods for the synthesis of selenium-containing heterocyclic derivatives has attracted much attentions. The recent progress in this rapidly growing area, including metal catalysis, electrochemical catalysis, visible-light catalysis, organocatalysis, and other selenocyclization types, is highlighted with an emphasis on the scope and the mechanisms of these different reactions.
\end{abstract}

Keywords organic selenide; heterocyclic compound; selenocyclization; catalysis

硫系化合物在有机合成中具有广泛的用途，由于它 们特殊的化学反应性、区域选择性和立体选择性, 已成 为极具吸引力的合成目标分子 ${ }^{[1]}$. 其中, 有机硒化合物 是合成化学中重要的中间体 ${ }^{[2]}$, 作为核心结构骨架存在 于数量可观的具有生物活性的药物分子中(图 1). 例如, 可以用作抗肿瘤药物 ${ }^{[3]}$ 、抗菌药物 ${ }^{[4]}$ 、抗增殖剂 ${ }^{[5]}$ 和抗菌 剂[ [6]等. 除此之外, 有机硒化合物也是材料化学中重要 的结构单元 ${ }^{[7]}$. 因此, 有机硒化合物在有机合成、药物及 有机材料等领域具有广泛的应用. 在碳链中引入硒原子
可以改变化学反应性以及药理性质和毒理性质, 就药理 性质而言，硒原子的存在可以显著提高底物的天然生物 活性 $^{[8]}$, 这主要归因于硒原子可以作为氢键受体或电子 供体, 改变酶活性位点的化学性质. 构建碳-硒键的方 法主要包括在有机分子中加入亲核、亲电或自由基硒试 剂 ${ }^{[9]}$, 通常在温和条件下, 高区域选择性地发生反应. 因此, 开发绿色新颖、高效实用的有机硒化合物的合成 方法引起化学家的广泛关注 ${ }^{[10]}$. 另一方面, 杂环化合物 是构成众多天然产物和药物分子的重要骨架 ${ }^{[11]}$, 随着合

\footnotetext{
* Corresponding author. E-mail: liuyj691@nenu.edu.cn

Received August 8, 2020; revised September 9, 2020; published online September 29, 2020.

Project supported by the Outstanding Youth Project of Natural Science Foundation of Heilongjiang Province (No. YQ2019B004), the General Project of Natural Foundation of Heilongjiang Province (No. H2017001), the Youth Innovation Talent Project of Harbin University of Commerce (Nos. 2016QN056, 2019CX38), the Youth Reserve Talent Program of Harbin University of Commerce (No. 2019CX36) and the Natural Foundation Co-pilot Project of Heilongjiang Province (Nos. LH2020H068, LH2020H070).

黑龙江省自然科学基金优秀青年项目(No. YQ2019B004)、黑龙江省自然基金面上目(No. H2017001)、哈尔滨商业大学青年创新人才(Nos. 2016QN056, 2019CX38)、哈尔滨商业大学青年后备人才(No. 2019CX36)和黑龙江省自然基金联合引导(Nos. LH2020H068, LH2020H070)资助项目.
} 
成化学家对于有机硒杂环衍生物合成和应用兴趣的与 日俱增, 相关成果近年来被广泛报道. 本文将就近年来 有机硒参与的环化反应按照金属催化、电化学驱动、可 见光驱动及有机分子催化等类型进行较为详细地介绍.

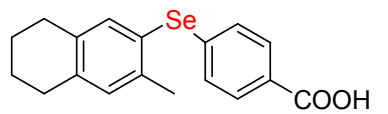

1

RAR agonist<smiles>Clc1ccc2c([Se]c3ccccc3)ccnc2c1</smiles>

3

Antioxidant<smiles>Nc1cc2c(ncn2C[Se]CCO)c(=O)[nH]1</smiles>
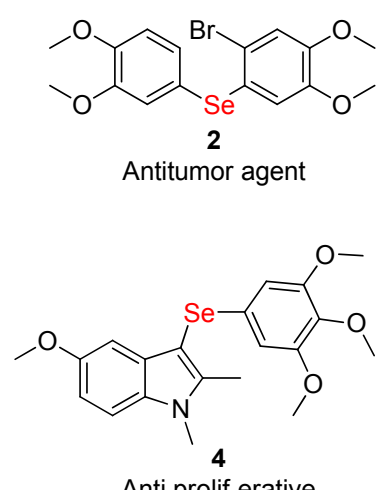

Anti prolif erative<smiles>O=c1c2ccccc2[se]n1-c1ccccc1</smiles>

Potent anticaner properties
图 1 含硒的杂环生物活性分子

Figure 1 Selenium-containing heterocyclic bioactive molecules

\section{1 金属催化}

长期以来, 过渡金属催化以较高的选择性提供各种 范围广泛的目标化合物 ${ }^{[12]}$. 随着过渡金属催化合成有 机硒化合物反应的发展, 采用铜或铁等催化体系, 已经 开发出高选择性的硫族化合物的转化 ${ }^{[13]}$.

2008 年, Zeni 课题组 ${ }^{[14]}$ 报道了一种简单而有效的 $\mathrm{CuI}$ 催化烯炔类化合物的环化反应, 成功地合成了多种 3-取代的硫系化合物(Scheme 1). 在最佳反应条件下, 硒烯炔与二芳基二硒醚反应，反应对具有不同给电子和 吸电子基团的底物 7 表现出极好的耐受性. 除此之外, 碲烯炔与二芳基二硒醚也反应良好, 但反应对芳环取基 的电子效应不敏感, 均以相似的产率合成了目标产物.

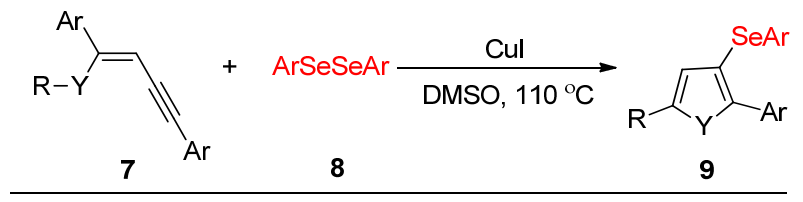

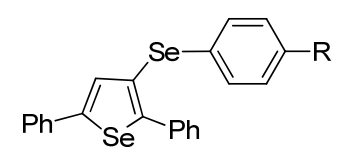

9a, $\mathrm{R}=\mathrm{CH}_{3}, 80 \%$

$9 b, \mathrm{R}=\mathrm{Cl}, 89 \%$

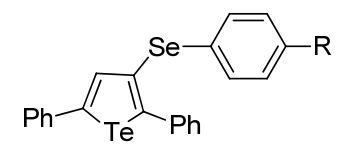

9c, $\mathrm{R}=\mathrm{CH}_{3}, 72 \%$

9d, $\mathrm{R}=\mathrm{Cl}, 85 \%$
图式 $1 \mathrm{CuI}$ 催化硒烯炔和碲烯炔的环化

Scheme 1 CuI catalyzed selenoenyne cyclization and telluroenyne cyclization
2009 年, 张兴国及其同事 ${ }^{[15]}$ 报道了在 $\mathrm{MeCN}$ 溶液 中, 以 $\mathrm{PdCl}_{2}$ 为催化剂, 2-炔基茴香醚与二硒醚发生环化 反应，得到了相应的苯并 $[b]$ 呋喃(Scheme 2). 其中, 2-炔 基茴香醚 10 与二芳基二硒醚以中等至优异的产率生成 了相应的 3-硒基苯并呋喃. 除此之外，二烷基二硒醚也 表现出了良好的反应性.

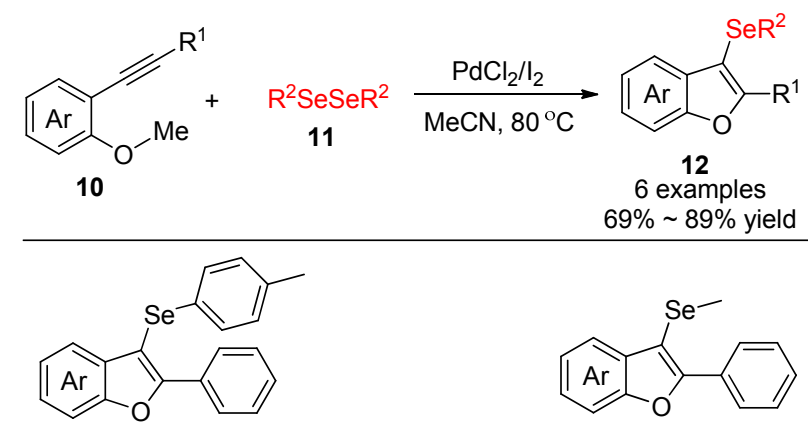

$12 a, 89 \%$

12b, $83 \%$

图式 2 钯催化炔基苯酚衍生物环化

Scheme 2 Palladium catalyzed cyclization of alkynylphenol derivatives

2010 年, Zeni 课题组 ${ }^{[16]}$ 报道了以廉价环保的 $\mathrm{FeCl}_{3}$ 为催化剂, 易得的二硒醚和 2-炔基茴香醚发生环化反 应, 生成了相应的苯并 $[b]$ 呋喃(Scheme 3 ). 令人满意的 是，二烷基二硒醚或二苄基二硒醚均在反应过程中表现 出了良好的反应活性. 除此之外，硒原子上芳环取代基 的电子效应对反应产率影响较大. 其中, 芳环上具有供 电子取代基的底物 14 以较高的产率得到目标产物, 但 具有 $\mathrm{CF}_{3}$ 或 $\mathrm{Cl}$ 基团的二芳基二硒醚的产率相对较差.

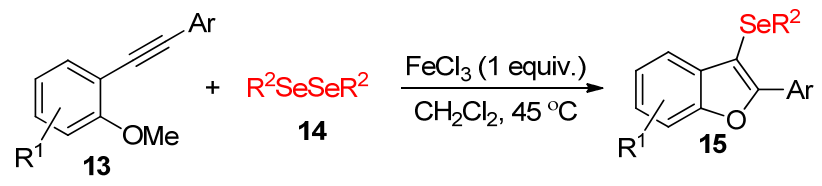

$\mathrm{R}^{1}=\mathrm{H}, \mathrm{Me}, \mathrm{F}$;

$\mathrm{Ar}=\mathrm{C}_{6} \mathrm{H}_{5}, p-\mathrm{MeC}_{6} \mathrm{H}_{4}, m-\mathrm{CF}_{3} \mathrm{C}_{6} \mathrm{H}_{4}$

17 examples<smiles>[R]c1ccc([Se]c2c(-c3ccccc3)oc3ccccc23)cc1</smiles>

15a, $\mathrm{R}=\mathrm{Cl}, 62 \%$

15b, $\mathrm{R}=\mathrm{CH}_{3}, 90 \%$<smiles>[R][Se]c1c(-c2ccccc2)oc2ccccc12</smiles>

15e, $\mathrm{R}=n-\mathrm{Bu}, 80 \%$ 15f, $\mathrm{R}=\mathrm{CH}_{2} \mathrm{Ph}, 64 \%$
图式 3 铁(III)催化炔基茴香醚的环化反应

Scheme 3 Iron(III) catalyzed cyclization of anisoles

2011 年, 周锡庚及其同事 ${ }^{[17]}$ 报道了 $\mathrm{CuI}$ 催化的 2炔基苯胺与二苯基二硒醚的硒胺化/环化反应，以令人 满意的产率合成了 3-硒基吲哚(Scheme 4). 与传统方法 相比，该方法具有简单、经济、高效和所使用的试剂环 境友好等特点. 


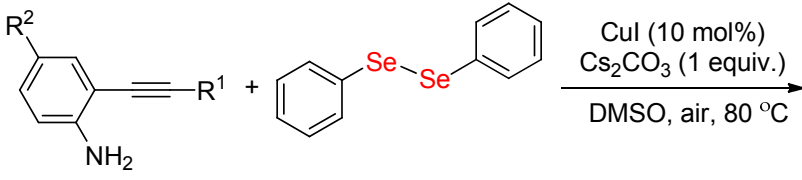

16

17

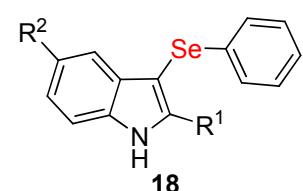<smiles>c1ccc([SeH]c2c(-c3ccccc3)[nH]c3ccccc23)cc1</smiles>

18a, $72 \%$<smiles>COCCc1[nH]c2ccccc2c1[SeH]c1ccccc1</smiles>

18b, $77 \%$<smiles>Clc1ccc2[nH]c(-c3ccccc3)c(S[Se]c3ccccc3)c2c1</smiles>

$18 c, 81 \%$
图式 $4 \mathrm{CuI}$ 催化 2-炔基苯胺的环化

Scheme 4 CuI catalyzed cyclization of 2-alkynylanilines

2013 年, Zeni 课题组 ${ }^{[18]}$ 开发了在铜(II)催化剂的作 用下高炔丙基硒化物的分子内环化反应, 合成了多种 4溴硒吩衍生物(Scheme 5). 在最佳反应条件下, 测试了 高炔丙基硒化物 19 的官能团耐受性, 其中芳环上具有 供电子和吸电子取代基的底物均表现出了良好的反应 活性，除此之外，取代基的空间效应对反应产率影响不 大.

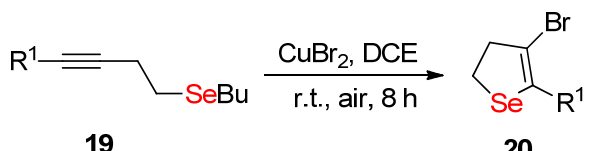

19

examples up to $72 \%$ yield

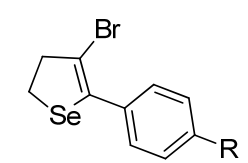

20a, $\mathrm{R}=\mathrm{OMe}, 62 \%$ 20b, $\mathrm{R}=\mathrm{Cl}, 60 \%$

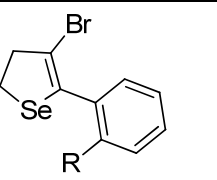

20c $\mathrm{R}=\mathrm{Me}, 60 \%$

20d, $\mathrm{R}=\mathrm{MeO}, 55 \%$
图式 5 铜(II)催化高炔丙基硒醚的环化

Scheme 5 Copper(II) catalyzed cyclization of high propargyl selenide

2014 年, 该课题组 ${ }^{[19]}$ 在以 $\mathrm{FeCl}_{3}$ 为催化剂的条件下, 以易得的芳基丙酸酯和芳基丙酰胺为原料分别和二硒 醚反应, 合成了 3-有机硒基- $2 H$-香豆素和 3-有机硒基喹啉酮类化合物(Scheme 6). 该方法反应条件温和, 且 二硒醚具有双重作用, 既可以作为环化剂, 又可以作为 碱来恢复芳香体系.

该作者通过对照实验进行了进一步的研究，提出了 如 Scheme 7 所示的可能的反应机理. 首先, 该反应通过 配合物 $\left[\mathrm{Fe}(\mathrm{SePh})_{2}\right] \mathrm{L}_{n}$ 初始活化底物 21 的碳碳参键形成

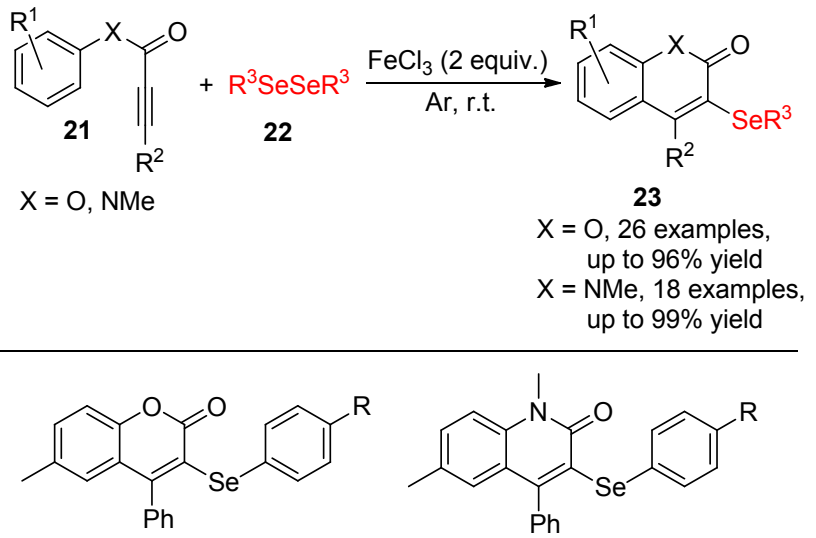

23a, $R=F, 91 \%$

23b, $\mathrm{R}=\mathrm{CH}_{3}, 94 \%$

23c, $\mathrm{R}=\mathrm{Cl}, 73 \%$

23d, $R=F, 84 \%$

23e, $\mathrm{R}=\mathrm{H}, 78 \%$

23f, $\mathrm{R}=\mathrm{CH}_{3}, 85 \%$

图式 6 铁盐催化芳基丙酸酯和芳基丙酰胺的环化

Scheme 6 Cyclization of arylpropionates and arylpropionamides catalyzed by iron salt

中间体 24. 芳环电子云对中间体 24 进行攻击产生环化 产物 25, 产物 25 还原消除得到稳定的碳正离子 26. 最 后，在硒酸盐阴离子的推动下，通过打破 $\mathrm{C}-\mathrm{H}$ 键来脱 氢恢复了芳香体系，得到了环化产物 23.
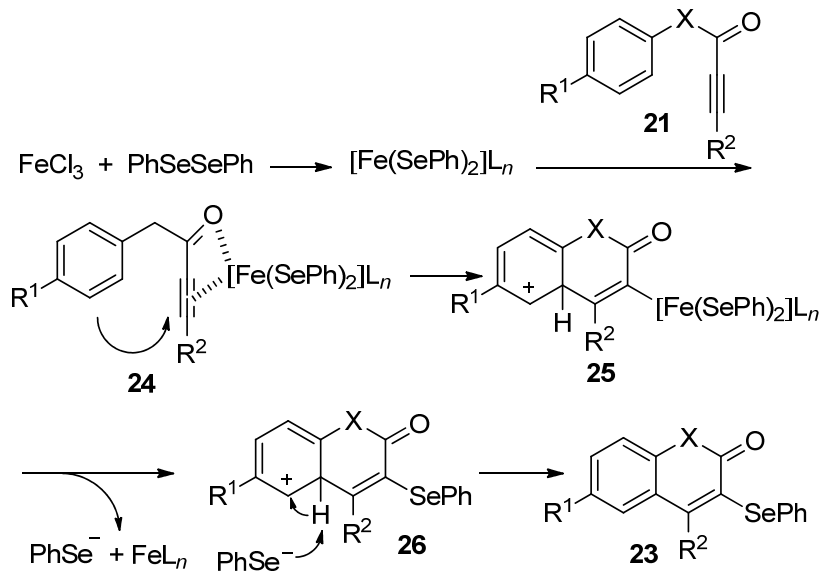

图式 7 铁盐催化芳基丙酸酯和芳基丙酰胺的环化的可能机 理

Scheme 7 Proposed mechanism of arylpropionates or arylpropionamides cyclization catalyzed by iron salt

同年，Ranu 等 ${ }^{[20]}$ 开发了一种通用而高效的硫系化 合物合成方法，使用廉价的 $\mathrm{CuO}$ 纳米颗粒代替昂贵的 $\mathrm{CuI}$ 作为催化剂, 以 1,3-二烯基溴化物为原料, 与硒氰 化钾通过简单的一锅反应，得到了新的硒苯类化合物 (Scheme 8). 在最佳反应条件下, 不同给电子和吸电子 基团取代的二烯基溴化物 27 都表现出良好的反应活性.

令人满意的是，在此条件下，不同芳基取代的 1,3二烯基一偕二溴化物 30 也可顺利地与硒氰化钾反应，得 到了硒基硒苯类化合物 31 (Scheme 9). 其中, 1,3,5-三烯 


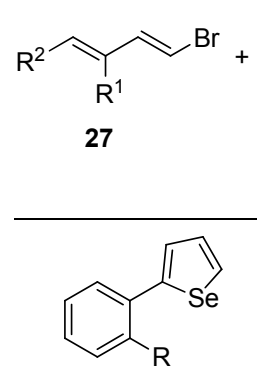

29a, $\mathrm{R}=\mathrm{NO}_{2}, 88 \%$

29b, $\mathrm{R}=\mathrm{OMe}, 93 \%$

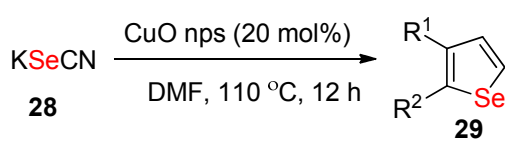

12 examples up to $95 \%$ yield

图式 $8 \mathrm{CuO}$ 催化 1,3-二烯基溴化物的环化

Scheme $8 \mathrm{CuO}$ catalyzed cyclization of 1,3-diene bromide

基一偕二溴化物可毫无困难地转化为硒基乙烯基取代硒 苯 31d, 值得注意的是，未观察到苯基二硒化物的形成.
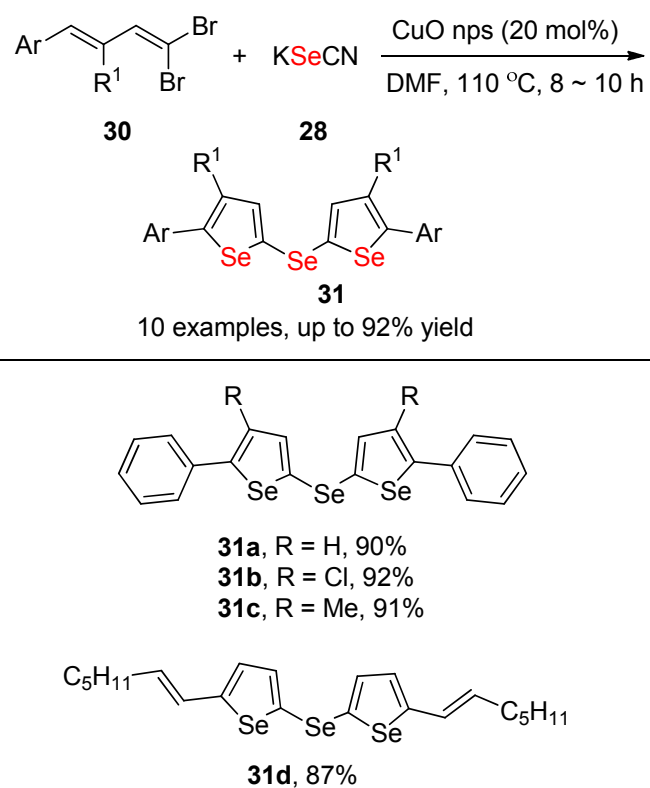

图式 $9 \mathrm{CuO}$ 催化 1,3-二烯基二溴化物的环化

Scheme $9 \mathrm{CuO}$ catalyzed cyclization of 1,3-diene dibromides

2015 年, Swamy 课题组 ${ }^{[21]}$ 报道了一种新颖、高效的 一锅反应. 该方法以 $[\mathrm{Cu}]$ 为催化剂, 以官能化的氮化物 为原料与单质硒发生环化反应, 得到相应的苯并 $[1,4,2]$ 噻吩嗪 1,1-二氧化物(Scheme 10). 这一方法可被扩展到 苯并二硫氮草类药物/苯并噻吩氮杂草类药物的合成, 证明了其在药物合成方面的用途.

2015 年, Zeni 及其同事 ${ }^{[22]}$ 实现了在 $\mathrm{FeCl}_{3}$ 催化体系 下，2-氨基芳基-2-炔醇与二硒醚的环化反应，区域选择 性地合成了 3-有机硒基喹啉(Scheme 11). 该反应对带不 同的给电子和吸电子基团的底物 36 和 37 均表现出良好 的耐受性，但二苠基二硒醚与 2-氨基芳基-2-炔醇反应 时没有得到预期产物, 这可能受苄基硒化物的低稳定性 的影响.<smiles>[R]C#CN([R])S(=O)(=O)c1ccc([R])cc1I</smiles>

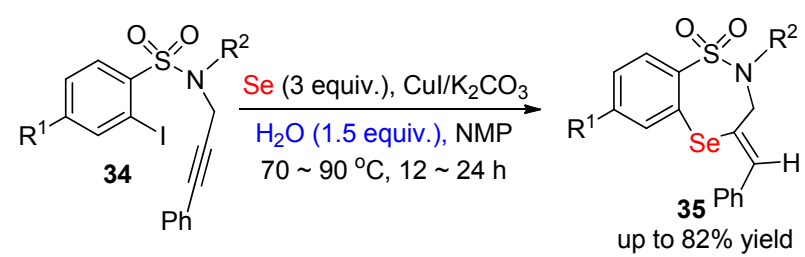

图式 10 铜催化官能化氮化物的环化

Scheme 10 Cyclization of functionalized nitrides catalyzed by copper

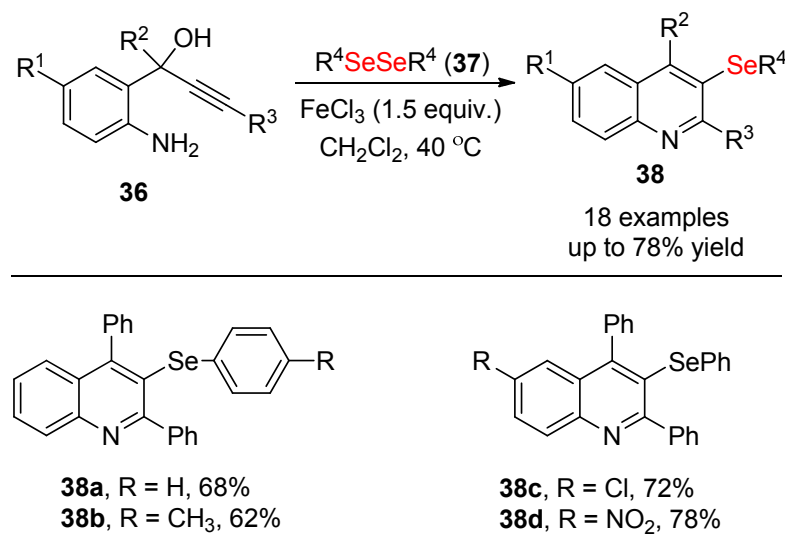

图式 11 铁(III)催化芳基炔醇的环化

Scheme 11 Iron(III) catalyzed cyclization of arylalkynol

2016 年, 徐政虎课题组 ${ }^{[23]}$ 报道了铜(I)催化的点击 反应，得到了 5-硒代三唑类化合物(Scheme 12), 该反应 在非常温和的条件下进行, 不需要配体, 具有较高的区 域选择性和良好的官能团兼容性.

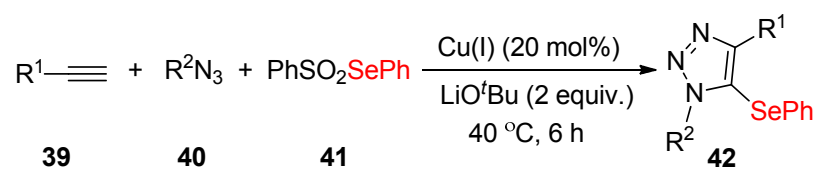

图式 12 铜(I)催化亲代硒化试剂合成三坐

Scheme 12 Synthesis of triazole by copper(I) catalyzed parent selenide

2018 年, Yasuike 及其同事 ${ }^{[24]}$ 开发了一种简单通用 三唑类化合物的合成方法，该方法以 $\mathrm{CuI}$ 为催化剂，在 1,10-邻菲咯啉存在下, 乙炔基二苯乙烯、有机叠氮化合 物和二芳基二硒醚发生三组分反应(Scheme 13). 在最 佳条件下, 炔烃 43 的取代基的电子性质均对反应活性 无影响, 并以中等至优异的产率得到 5-硒基-1,2,3-三唑. 在对照实验的基础上，提出了该三组分反应的可能机 


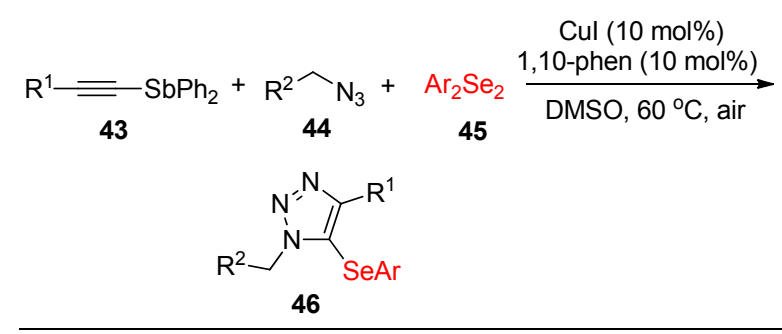

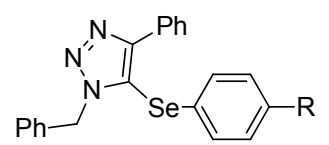

46a, $\mathrm{R}=\mathrm{Me}, 87 \%$

46b, $\mathrm{R}=\mathrm{Cl}, 71 \%$

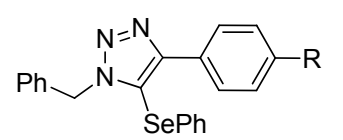

46c, $\mathrm{R}=\mathrm{Me}, 73 \%$

46d, $\mathrm{R}=\mathrm{CF}_{3}, 73 \%$
图式 13 铜(I)催化合成 5-硒基-1,2,3-三唑

Scheme 13 Copper(I) promoted formation of 1,2,3-triazoles

理(Scheme 14). 该反应涉及在 $\mathrm{CuI}$ 催化下乙炔基苯乙烯 43 和叠氮化物 44 生成 5-二苯乙烯三唑 $47^{[25]}$. 首先, 二 芳基二硒醚 45 与双金属配合物 $\left[(\mathrm{phen})_{2} \mathrm{CuI}_{2}\right](48)$ 反应生 成 $\mathrm{Cu}^{\mathrm{III}}$ 中间体 $49^{[26]}$. 然后, 化合物 47 在三氮唑的 5-位 上攻击中间体 49 形成中间体 $\mathbf{5 0}$, 中间体 50 经过还原消 除得到中间体 51, 并再生铜配合物. 芳基硒化物阴离子 攻击锑产生 5-硒酰三唑 46 和 $\mathrm{Ph}_{2} \mathrm{SbSeAr}$, 在空气中水解, 转化为硒醇 52 和 $\mathrm{Ph}_{2} \mathrm{SbOH}$, 硒醇通过空气氧化转化为 二硒醚.

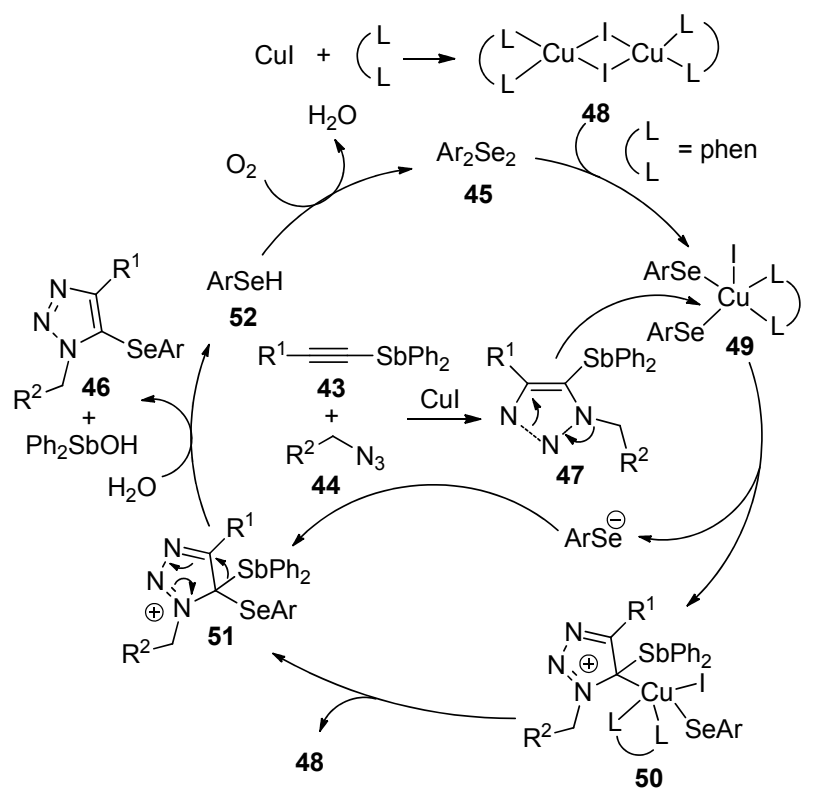

图式 14 铜(I)催化生成 1,2,3-三唑的可能机理 Scheme 14 Possible mechanism of copper(I) promoted formation of 1,2,3-triazoles

2017 年, Zeni 课题组 ${ }^{[27]}$ 研究了 $\mathrm{CuI}$ 催化的炔丙基吡 啶与二硒醚化合物的环合反应，合成了 2-(有机硒基)吲哚嗪类化合物(Scheme 15). 在最佳条件下，二硒醚芳 环上供电子取代基的位置对反应有一定影响, 与芳环相
连的邻位甲基降低了反应活性. 除此之外，当二丁基二 硒醚作为硒源时，由于 $\beta$-硒氧化物的消除，没有检测到 所需的产物.

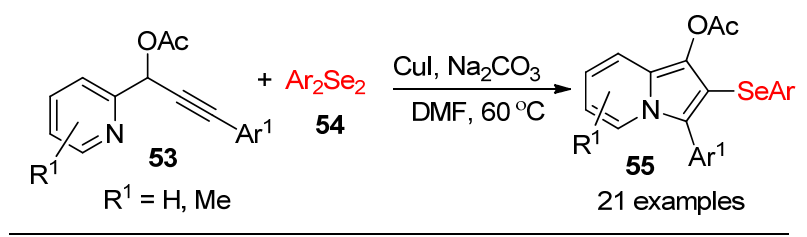

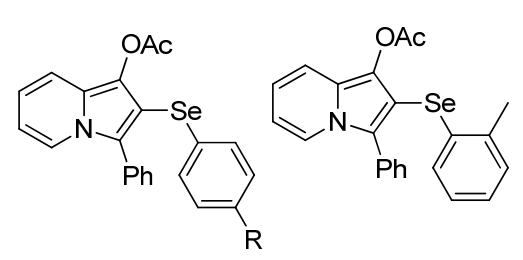

55a, $\mathrm{R}=\mathrm{CH}_{3}, 72 \%$ 55b, $\mathrm{R}=\mathrm{Cl}, 84 \%$

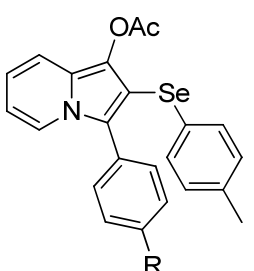

55d, $R=O M e, 75 \%$ 55e, $\mathrm{R}=\mathrm{Cl}, 63 \%$
图式 15 铜(I)催化形成吲哚嗪

Scheme 15 Copper(I) catalyzed formation of indolizines

2017 年, Godoi 及其同事 ${ }^{[28]}$ 以二甲基亚砜为溶剂, 在常温、常压和无碱条件下，实现了 2-炔基苯酚与二硒 醚的分子内环化反应，合成了 3-有机硒基-苯并 $[b]$ 呋喃 (Scheme 16). 该反应对具有不同给电子和吸电子基团 的底物 56 均表现出极好的耐受性. 除此之外, 还考察了 二烷基二硒醚在反应过程中的活性，但产物 $\mathbf{5 8 d}$ 和 $\mathbf{5 8 e}$ 产率不佳.

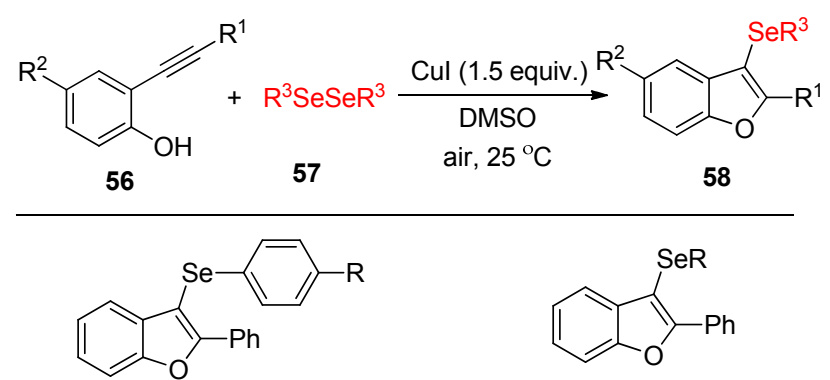

58a, $R=F, 57 \%$

58b, $\mathrm{R}=\mathrm{Cl}, 61 \%$

58c, $\mathrm{R}=\mathrm{Me}, 63 \%$

58d, $\mathrm{R}=\mathrm{Bu}, 30 \%$

58e, $\mathrm{R}=\operatorname{Pr}, 32 \%$

图式 $16 \mathrm{CuI}$ 催化炔基苯酚的环化

Scheme 16 CuI catalyzed cyclization of alkynylphenol

2018 年, Zeni 课题组 ${ }^{[29]}$ 报道了在铜(I)催化条件下, 以二甲基亚砜为溶剂，以丁基硒基炔丙醇为底物与二硒 醚反应合成 3-(有机硒基)硒苯类化合物(Scheme 17). 其 中，底物 59 的取代基的电子效应和空间效应均对产率 有影响, 其中丁基硒基炔丙醇中存在未取代的苯基时, 以较高的产率合成了 60c. 然而，炔丙基上取代芳基的 存在对产率有负面影响. 


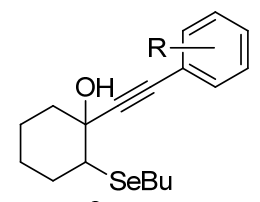

59

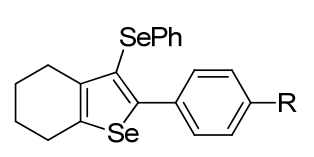

60a, $\mathrm{R}=\mathrm{OMe}, 70 \%$ 60b, $\mathrm{R}=\mathrm{Br}, 57 \%$

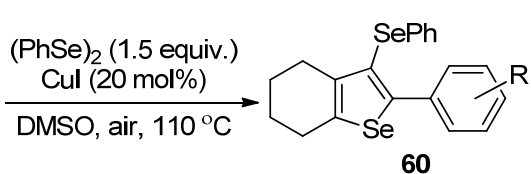
6 examples

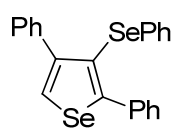

$60 c, 78 \%$

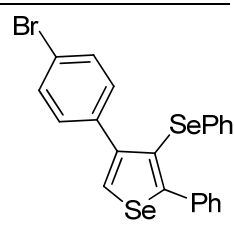

60d, $29 \%$

图式 17 铜催化炔丙醇的环化

Scheme 17 Copper catalyzed cyclization of propargyl alcohol

2018 年, 徐燕丽及其同事 ${ }^{[30]}$ 以丙醇酸、二硒醚和叠 氮化物为原料, 开发了一种简单有效的铜催化脱羧/点 击反应，用于合成 1,4-二取代 5-芳基硒基-1,2,3-三唑 (Scheme 18), 该反应对芳环的电子效应和空间效应均 表现出极好的耐受性. 除此之外, 用噻唑蓝(MTT)法检 测了多取代产物的体外抗癌活性, 其中化合物 64a, 64b 和 64c 显示出有效的癌细胞生长抑制活性. 该催化体系 具有反应条件温和、操作简便、效率高、区域选择性高 及与空气相容性好等特点.

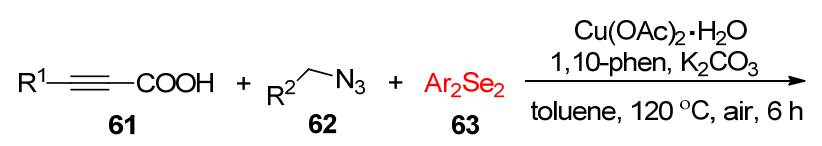

$$
\underbrace{\mathrm{R}^{1}}_{61 \% \sim 86 \%}
$$<smiles>Fc1ccc(-c2nnn(Cc3ccccc3)c2Sc2ccccc2)cc1</smiles>

$64 a$<smiles>FC(F)(F)c1ccc(-c2nnn(Cc3ccccc3)c2Oc2ccccc2)cc1</smiles>

64b

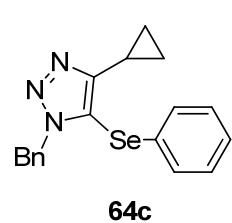

$64 c$

图式 18 乙酸铜(II)促进 1,2,3-三坐的生成

Scheme 18 Copper(II) acetate-promoted formation of 1,2,3triazoles

为了探索可能的反应途径, 进行了对照实验, 并提 出了如 Scheme 19 所示的反应机理. 结果表明, $\mathrm{K}_{2} \mathrm{CO}_{3}$ 在脱除丙酸中的酸性氢方面起着重要作用, 并证明了 $\mathrm{O}_{2}$ 在反应中起着重要作用. 首先, 通过配位作用 $1,10-$ 邻菲咯啉和 $\mathrm{Cu}(\mathrm{OAc})_{2} \cdot \mathrm{H}_{2} \mathrm{O}$ 形成活性铜(II)中间体 $\mathbf{6 5}$, 然 后与炔酸 61a 反应生成铜(II)中间体 66. 66 通过脱羧反 应产生铜(II)中间体 67, 并释放一分子 $\mathrm{CO}_{2} .67$ 与二苯二 硒醚反应生成中间体 68 , 经过还原消除得到苯基(苯乙 炔基) - 硒烷 69 以及 70,70 与 69 和 $62 \mathrm{a}$ 络合生成中间体

71, 经历氧化环化形成 72, 72 的还原消除提供了所需的 产物 64 和铜(I) 70 , 最后在空气气氛下将 70 转化为 65 完成反应.

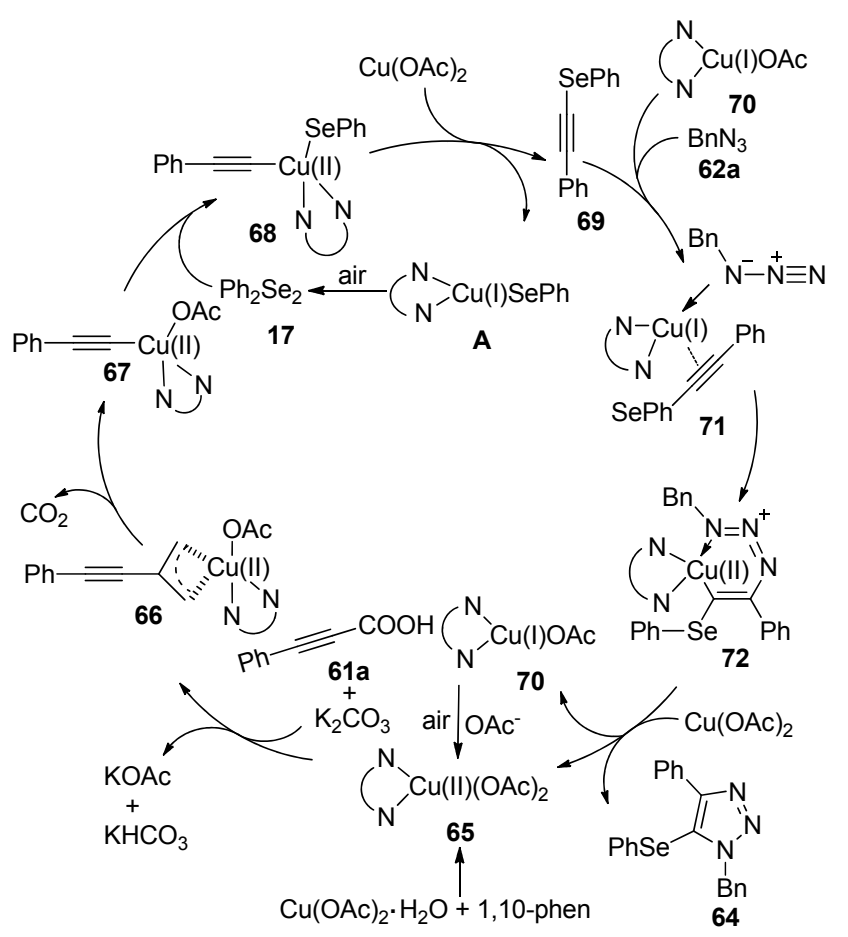

图式 19 乙酸铜(II)促进 1,2,3-三唑的生成的机理 Scheme 19 Mechanism of copper(II) acetate-promoted formation of 1,2,3-triazoles

2019 年, 刘妙昌课题组 ${ }^{[31]}$ 报道了在 $\mathrm{AgNO}_{2}$ 催化条 件下, 2-炔基茴香醚(或 2 炔基硫代茴香醚)、硒粉和芳基 嗍酸进行自由基环化反应，成功地合成了相应的硒化苯 并呋喃(或苯并噻吩). 一系列具有不同给电子和吸电子 基团的底物均反应良好(Scheme 20), 但含硝基和甲氧

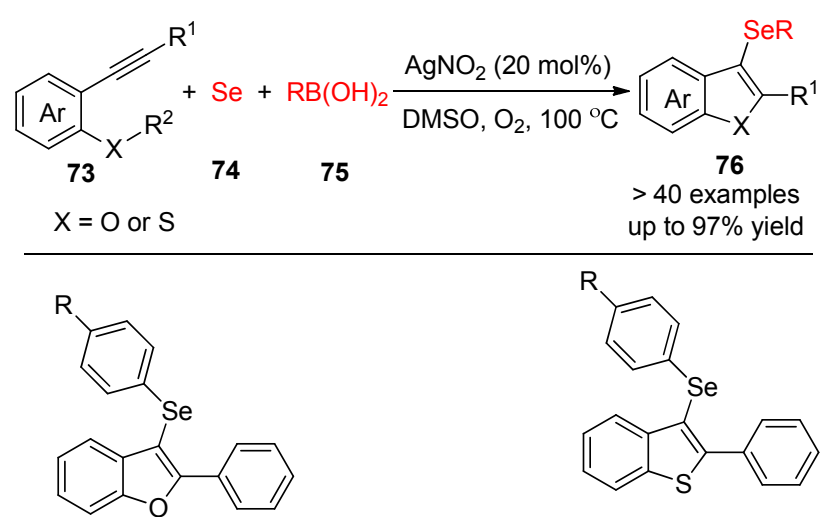

76a, $R=F, 91 \%$ 76b, $\mathrm{R}=\mathrm{Cl}, 97 \%$

76c, $R=F, 93 \%$ 76d, $\mathrm{R}=\mathrm{Cl}, 90 \%$

图式 $20 \mathrm{AgNO}_{2}$ 催化炔基茴香醚的环化

Scheme $20 \quad \mathrm{AgNO}_{2}$ catalyzed cyclization of anisole 
基等较强电子效应的取代基的底物 73 没有得到预期的 产物.

为了深入了解反应机理, 进行了针对芳基嗍酸、2炔基茴香醚以及硒粉的对照实验, 在标准反应体系中加 入自由基捕获剂四甲基哌啶氧化物(TEMPO)后，结果表 明反应过程可能涉及自由基途径. 根据这些结果和相关 文献 ${ }^{[32]}$, 提出了如 Scheme 21 所示的可能途径, 首先, 在 $\mathrm{AgNO}_{2} / \mathrm{O}_{2}$ 催化体系中, $\mathrm{ArB}(\mathrm{OH})_{2}$ 被氧化成芳基自由 基 77. 然后，该芳基自由基被硒粉捕获，生成芳基硒自 由基 78, 该自由基与 2-炔基茴香醚(或 2-炔基硫代茴香 醚)进行自由基加成，得到中间体 79. 最后，中间体 79 经过环合反应得到所需的产物，同时甲基自由基被转化 为甲醛。

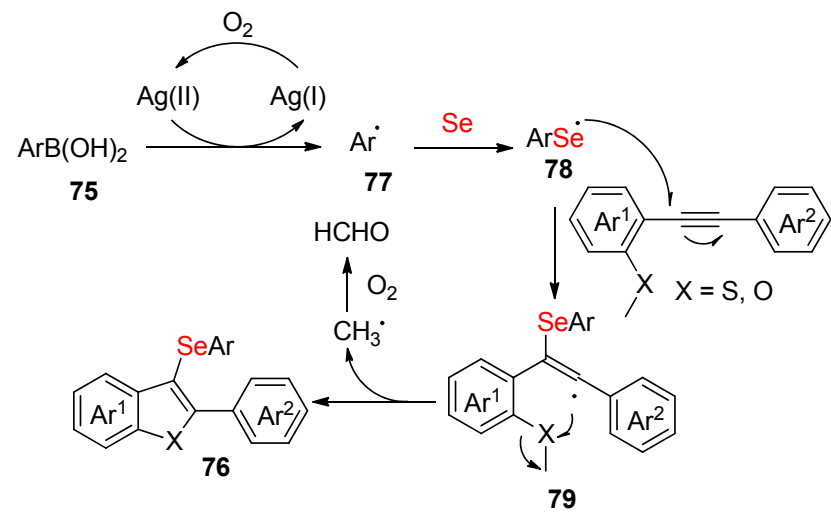

图式 21 自由基串联环化的可能途径

Scheme 21 Possible way of free radical cascade cyclization

2019 年, 孙凯及其同事 ${ }^{[33]}$ 报道了在无外部氧化剂 的条件下，使用现成的二硒醚作为硒源，进行铜催化的 硒代二氟甲基化反应，合成了多种 4-硒基取代的 $\alpha, \alpha$-二 氟- $\gamma$-内酰胺类化合物(Scheme 22). 一系列具有不同给 电子和吸电子基团的底物均反应良好, 以中等到良好的 产率生成了所需的产物, 但以具有 $N$-环戊基取代基的 溴二氟乙酰胺为底物时, 没有观察到所需的环化产物.
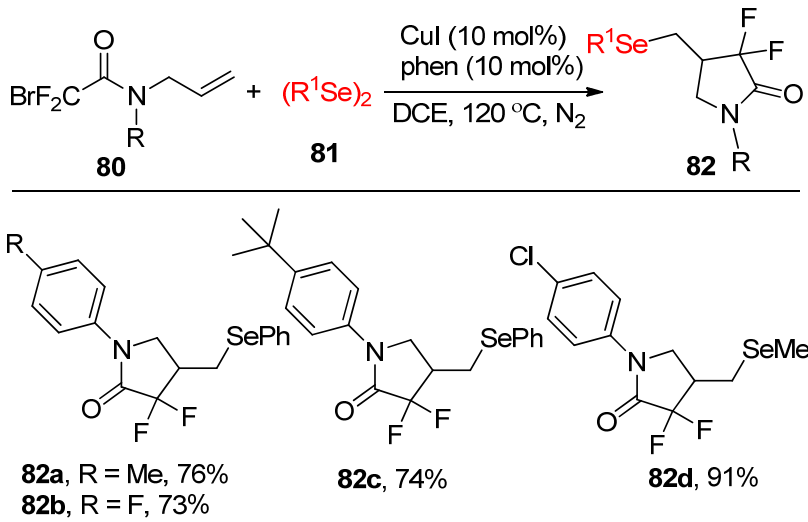

图式 22 铜催化烯烃的硒二氟甲基化反应

Scheme 22 Copper-catalyzed selenodifluoromethylation of alkenes

1018
在对照实验的基础上，作者提出了如 Scheme 23 所 示的可能的串级环化机理. 首先, $\mathrm{Cu}(\mathrm{I})$ 和底物 80 通过单 电子转移(SET)反应生成 $\mathrm{Cu}(\mathrm{II})$ 和自由基中间体 83 . 接 下来，通过将氟烷基加到不饱和双键上，快速进行外环 化反应，得到烷基中间体 84. 由于二硒醚是一种很好的 自由基捕获剂，最可能的途径是二硒醚捕获自由基中间 体 84, 生成 82 和硒基自由基, 进一步将 $\mathrm{Cu}(\mathrm{II})$ 还原为 $\mathrm{Cu}(\mathrm{I})$ 和硒基阴离子.

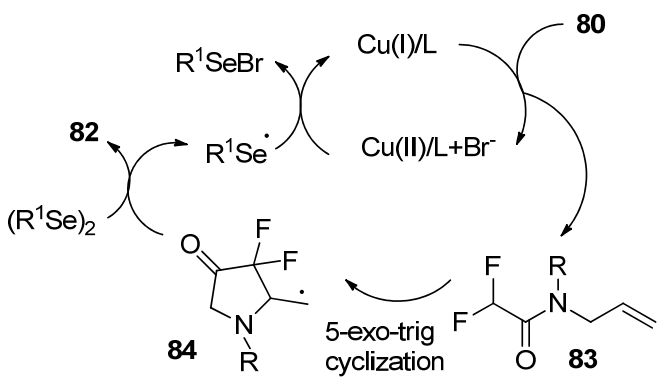

图式 23 硒二氟甲基化反应的可能机理 Scheme 23 Proposed mechanism of selenodifluoromethylation

2019 年, 李玮课题组 ${ }^{[34]}$ 报道了一例 $\beta, \gamma$-不饱和烯基 腙化合物与二硒化合物在醋酸铜催化下进行的硒胺基 化反应，合成了一系列的硒化吡唑啉类化合物(Scheme 24). 其中，底物 85 苯环上取代基的电子效应对反应没 有明显影响，均以非常好的收率获得目标产物. 该反应 的反应条件温和且不需要额外的氧化剂, 特别重要的是 该反应放大到克级规模时依然可以高效地进行.

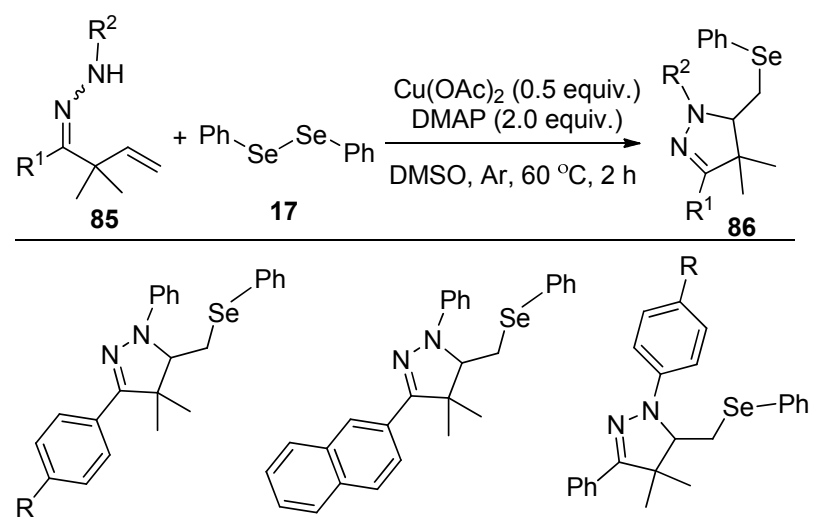

86a, $\mathrm{R}=\mathrm{MeO}, 93 \%$ 86b, $\mathrm{R}=\mathrm{Br}, 88 \%$

$86 c, 87 \%$

86d, $\mathrm{R}=\mathrm{CH}_{3}, 93 \%$ 86e, $\mathrm{R}=\mathrm{Cl}, 89 \%$

图式 24 铜催化 $\beta, \gamma$-不饱和烯基腙和二苯基二硒醚的硒胺基 化反应

Scheme 24 Copper-catalyzed sulfenoamination of $\beta, \gamma$-unsaturated hydrazones with diphenyl diselenide

2019 年，周锡庚等 ${ }^{[35]}$ 发展了一种以 $\mathrm{Pd}(\mathrm{OAc})_{2} / \mathrm{CuI}$ 为催化剂, 分步催化 2-(2,2-二溴乙烯基)胺、苯硽酸和联 硒化合物发生偶联/环化反应，合成 3-硒基吲哚化合物 的新方法(Scheme 25). 底物 87 中不同的官能团，如甲 
氧基、氯等在此反应中都能很好地兼容, 产物产率适中 到良好.

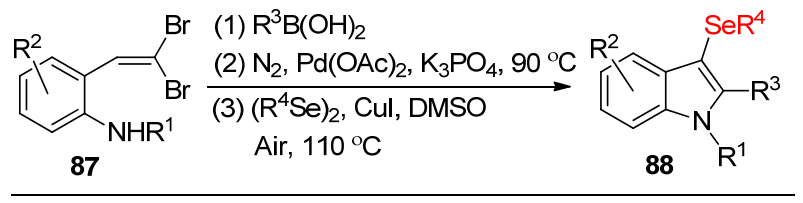<smiles>[R]n1c(-c2ccccc2)c([SeH2])c2ccccc21</smiles>

88a, $R=B n, 76 \%$ 88b, $\mathrm{R}=\mathrm{Ph}, 83 \%$<smiles></smiles>

$88 c, 87 \%$<smiles>Clc1ccc2[nH]c(-c3ccccc3)c(-c3ccccc3)c2c1</smiles>

88d, $73 \%$
图式 $25 \mathrm{Pd}(\mathrm{OAc})_{2} / \mathrm{CuI}$ 催化的二溴乙烯基 $)$ 胺与二硒醚的环化 Scheme 25 Cyclization of dibromovinyl amine and diselenide catalyzed by $\mathrm{Pd}(\mathrm{OAc})_{2} / \mathrm{CuI}$

\section{2 可见光驱动的硒环化}

近年来, 利用可见光催化环化反应已经开始普及, 这种反应可以在温和的条件下促进有机合成转化, 在这 一过程中可以避免使用过量的金属或非金属催化剂 ${ }^{[36]}$. 最近, 在没有光氧化还原催化剂的体系中进行的可见光 照射下的有机合成转化已经引起了相当大的关注, 这将 节省更多的金属或有机染料 ${ }^{[37]}$. 因此, 通过新的技术途 径实现可见光诱导的有机合成反应是非常可取的.

2013 年, Ragains 课题组 ${ }^{[38]}$ 报道了室温下可见光促 进的烯烃的硒环化反应. 该反应底物范围广, 不仅以较 高产率得到了含 $\mathrm{O}$ 杂环, 而且以中等产率得到了含 $\mathrm{N}$ 杂 环(Scheme 26). 该方案省去了硫族湿敏亲电材料的制 备，且不需要惰性气氛，具有操作简单等特点.
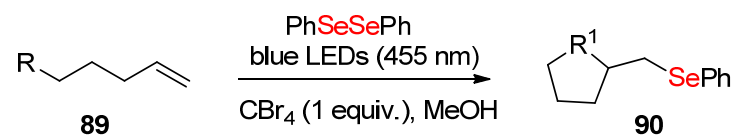

$$
\mathrm{R}=\mathrm{OH}, \mathrm{BocHN}, \mathrm{MeO}_{2} \mathrm{CHN} \quad \mathrm{R}^{1}=\mathrm{O}, \mathrm{BocN}, \mathrm{CO}_{2} \mathrm{MeN}
$$

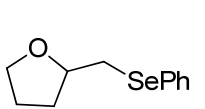

90a, $94 \%$

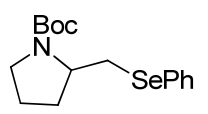

$90 b, 62 \%$

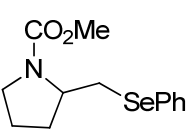

$90 c, 78 \%$
图式 26 光诱导促进烯烃环化

Scheme 26 Light-induced promotion of olefins cyclization

2017 年, 王否及其同事 ${ }^{[39}$ 报道了在 450 455 $\mathrm{nm}$ 的 可见光下, 不需要额外的光催化剂, 2-炔基苯胺与二硒 醚的一锅化氧化环化反应(Scheme 27). 值得注意的是, $N$-Ts-2-(环丙基乙炔基)苯胺对二芳基二硒醚 92 表现出 较高的反应活性, 以较高产率生成相应的环化产物. 但 是，底物 91 与二苯基二硒醚反应时，没有观察到二苯基
二硒醚的任何反应性.

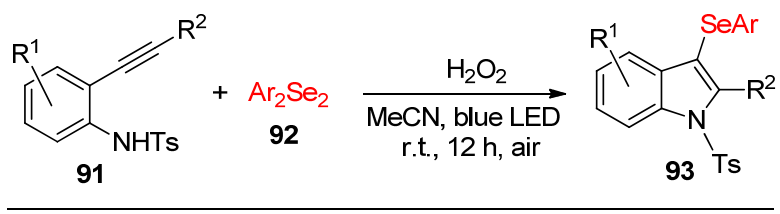<smiles>[R][Se]c1c(-c2ccccc2)n([AsH])c2ccccc12</smiles>

93a, $\mathrm{R}=\mathrm{Ph}, 97 \%$

93b, $\mathrm{R}=p-\mathrm{MeC}_{6} \mathrm{H}_{4}, 92 \%$ 93c, $\mathrm{R}=p-\mathrm{ClC}_{6} \mathrm{H}_{4}, 79 \%$<smiles>[R]c1ccc2c(c1)c([Se]c1ccccc1)c(-c1ccccc1)n2[As]</smiles>

93d, $\mathrm{R}=\mathrm{Me}, 92 \%$ 93e, $\mathrm{R}=\mathrm{Cl}, 76 \%$
图式 27 光诱导促进硒环化

Scheme 27 Photoinduced promotion of selenium cyclization

同年，王华课题组 ${ }^{[40]}$ 报道了通过可见光诱导合成 3苯硒基取代氮螺 [4.5] 三烯酮的方案(Scheme 28). 在非常 温和的反应条件下, 以 E $\sin \mathrm{Y}$ 为光催化剂, 使用 $N$-(对 甲氧基芳基)丙酰胺与二硒醚作为底物发生硒化-环化 反应，产率达 $63 \%$.

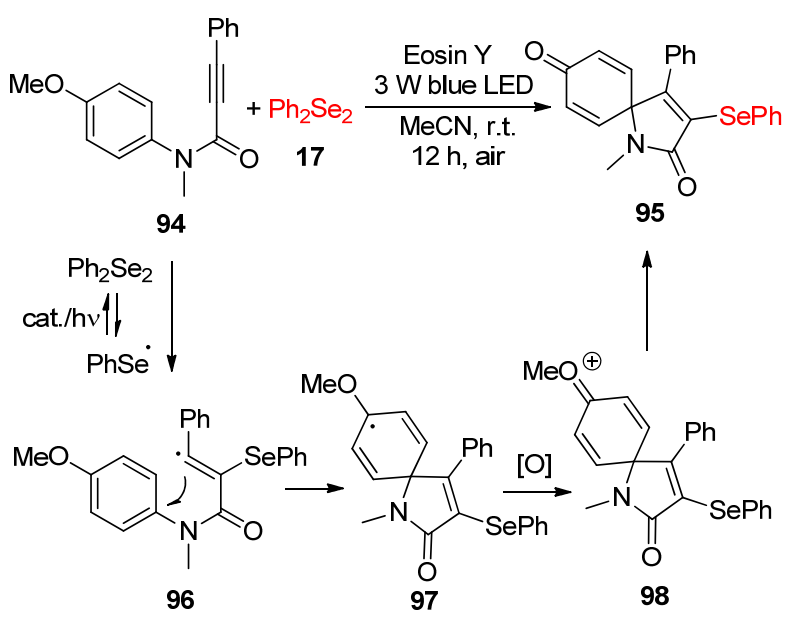

图式 28 蓝光下曙红 Y 存在下形成螺环化合物

Scheme 28 Formation of spiro-compounds in the presence of eosyn Y under blue light

该反应是通过级联自由基加成和环化过程实现炔 烃的去功能化，通过硒基与反应物相互作用产生乙烯基 自由基 96, 然后，乙烯基自由基发生分子内螺旋环化得 到 97. 自由基 97 是环化的产物, 在空气中氧化形成相 应的氧中间体 98, 最终, 该中间体转化为目标产物 95 .

2018 年, 宋秋玲及其同事 ${ }^{[41]}$ 报道了可见光催化的 甲基(2-(苯乙炔基)苯基)硒烷与磺酰氯发生级联环化反 应(Scheme 29), 芳环上具有供电子和吸电子基团的底 物 99 均以较好产率生成了苯并硒基苯类化合物. 除此 之外，该方法不需要过渡金属或添加剂，操作简单，条 
件温和，具有良好的官能团耐受性.
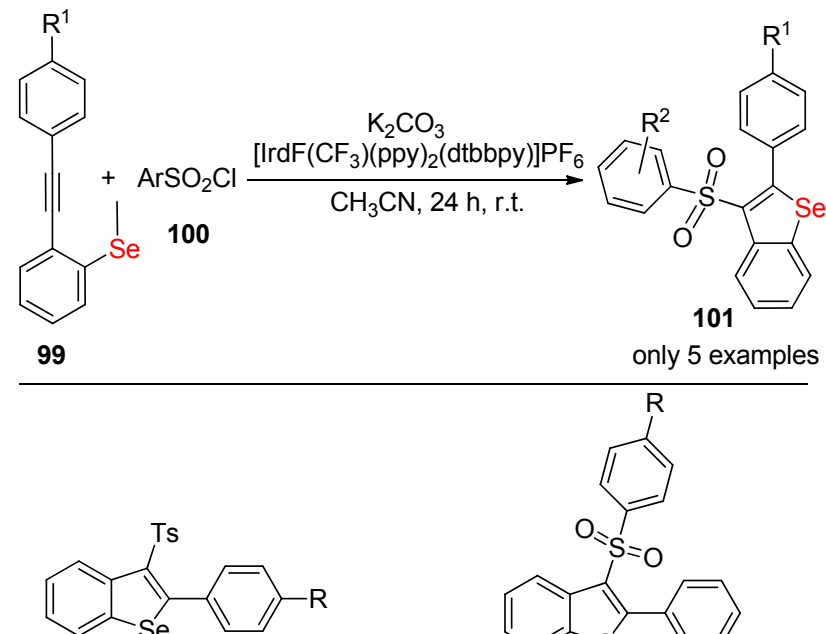

$$
\begin{aligned}
& \text { 101a, } \mathrm{R}=\mathrm{Br}, 75 \% \\
& \text { 101b, } \mathrm{R}=\mathrm{CH}_{3}, 67 \%
\end{aligned}
$$

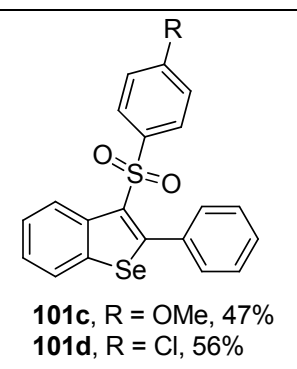

图式 29 光催化合成苯并硒基苯类化合物

Scheme 29 Photocatalytic synthesis of benzoselenylbenzenes

2018 年, Baidya 课题组 ${ }^{[42]}$ 介绍了一种制备硒取代的 螺环 [4.5] 三烯酩的有效方法(Scheme 30), 在没有外加光 催化剂的常温氧气气氛下, 通过可见光诱导 $N$-芳基烷 基酰胺与二芳基二硒醚发生自由基环化反应. 在最佳条 件下，二硒醚和底物 102 反应，均以中等到优良的产率 获得目标产物. 该方法具有操作简单、可扩展性强、底 物范围广及官能团兼容性好等特点.

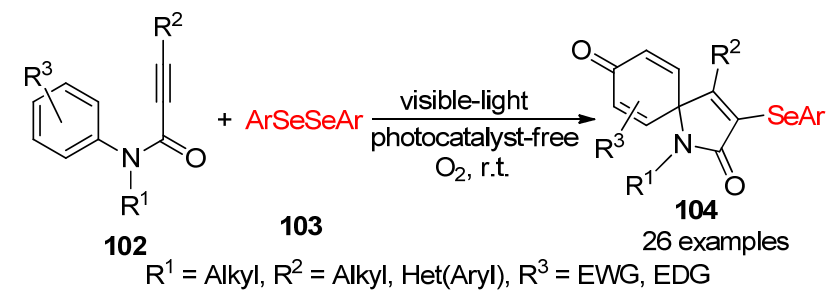<smiles>[R]C1=CC2(C=CC1=O)C(c1ccccc1)=C([Se]c1ccccc1)C(=O)N2C</smiles>

104a, $\mathrm{R}=\mathrm{Me}, 82 \%$ 104b, $\mathrm{R}=\mathrm{Br}, 73 \%$

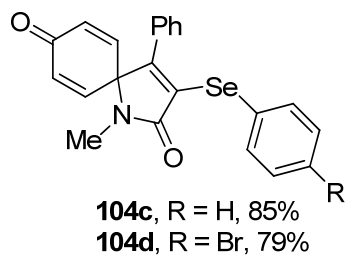

图式 30 光诱导促进合成三烯酮

Scheme 30 Photo-induced synthesis of triketene

为了确定该反应是否涉及自由基途径, 在标准反应 体系中加入 TEMPO 或 2,6-二叔丁基-4-甲基苯酚(BHT), 结果表明反应过程可能涉及自由基途径, 通过向相关底 物中加入过氧化叔丁醇(TBHP) 的结果, 进一步证实了 该反应涉及自由基途径。

基于这些发现, 提出了一个合理的反应机理(Sche- me 31), 蓝色 LED 照射时二芳基二硒醚产生的芳基硒自 由基与 102 生成乙烯基团 105, 随后进行分子间自由基 自环化得到 106. 在氧气气氛和二芳基二硒醚存在下, 中间体 106 转化为 107 , 最终在 $\mathrm{O}-\mathrm{O}$ 键断裂后生成螺 环化产物 104, 此机理与之前报道的先例一致 ${ }^{[43]}$.

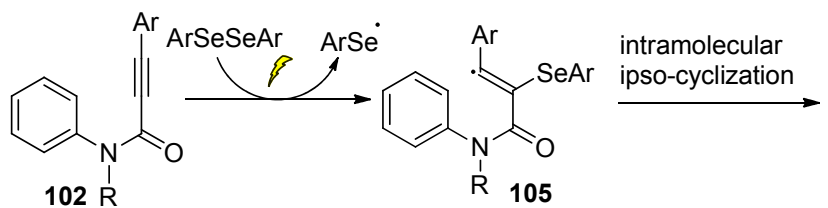

$102 \mathrm{R}$

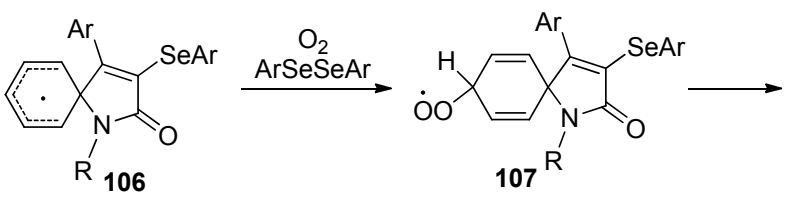<smiles>[R4]N1C(=O)C([SeH])=C(Br)C12C=CC(=O)C=C2</smiles>

图式 31 光诱导促进合成三烯酮的机理

Scheme 31 Mechanism of photo-induced synthesis of triketene

2019 年，潘英明及其同事 ${ }^{[44]}$ 开发了一种简单高效 的方法, 在 $40{ }^{\circ} \mathrm{C}, 25 \mathrm{~W}$ 白光照射下, 可见光诱导的炔 系环己二烯酮、二硒醚和水发生多组分串联环化反应 (Scheme 32). 该反应在环己酮骨架中同时引入硒基和 羟基，以级联方式形成三个新的立体中心. 除此之外, 用 MTT 法检测了产物的体外抗癌活性，发现化合物 $110 \mathrm{a}$ 和 $110 \mathrm{~b}$ 具有较强的肿瘤细胞生长抑制活性.

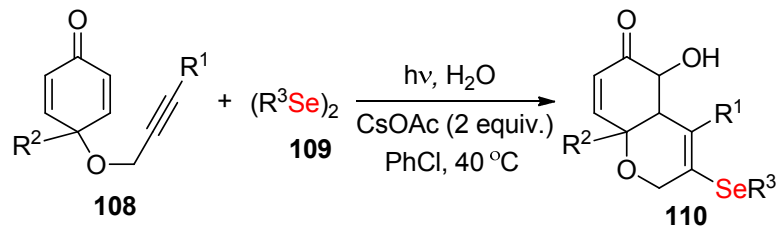

23 examples up to $88 \%$ yield<smiles>CC12C=CC(=O)C(O)C1C(c1ccc(-c3ccccc3)cc1)=C([Se]c1ccccc1)CO2</smiles>

$110 a$<smiles>CC12C=CC(=O)C(O)C1C(c1ccc3ccccc3c1)=C([Se]c1ccccc1)CO2</smiles>

$110 \mathrm{~b}$

图式 32 光诱导的炔系环已二烯酮与二硒醚环化 Scheme 32 Photoinduced cyclization of acetylenic cyclohexadienones and diselenides

为了进一步阐明反应机理，向标准体系中加入 TEMPO, 发现该过程受到抑制，表明这一转化过程中 可能涉及自由基途径. 根据实验结果和相关报道 ${ }^{[45]}$, 提 出了如 Scheme 33 所示的可能机理. 首先, 二苯基二硒 
醚在光照下产生苯硒基自由基 $\mathbf{1 1 1}$, 然后, 底物 $\mathbf{1 0 8}$ 进行 自由基加成得到烯基 112. 生成的烯基参与分子内自由 基反应生成中间体 113, 中间体 113 被另一种苯硒基自 由基捕获得到产物 114, 最终, 114 在 $\mathrm{CsOAc}$ 存在下与水 进行亲核取代, 得到所需的产物.

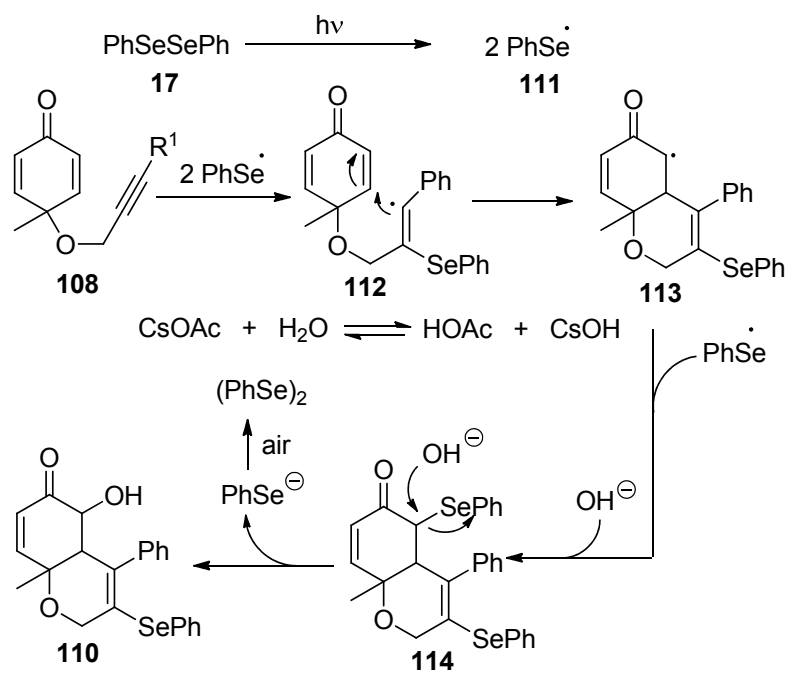

图式 33 光诱导的炔系环已二烯酮与硒环化的机理 Scheme 33 Mechanism of photoinduced cyclization of acetylenic cyclohexadienone and selenium

2019 年, 刘强课题组 ${ }^{[46]}$ 报道了在可见光照射下, 分 子氧为氧化剂的条件下, 通过烯烃与二硒醚的脱氢环化 反应构建杂环化合物(Scheme 34). 在最佳反应条件中, 没有外加光催化剂的情况下, 二氢异噁唑的产率为 $73 \%$, 但 4CzIPN 作为光催化剂可以促进反应进程. 除此 之外, 许多取代的烯丙基酰胺和二硒醚在此转化过程中 具有良好的官能团耐受性, 并以较好的产率得到了相应 的产物. 这种无过渡金属催化的方案具有原子经济性高 等特点.
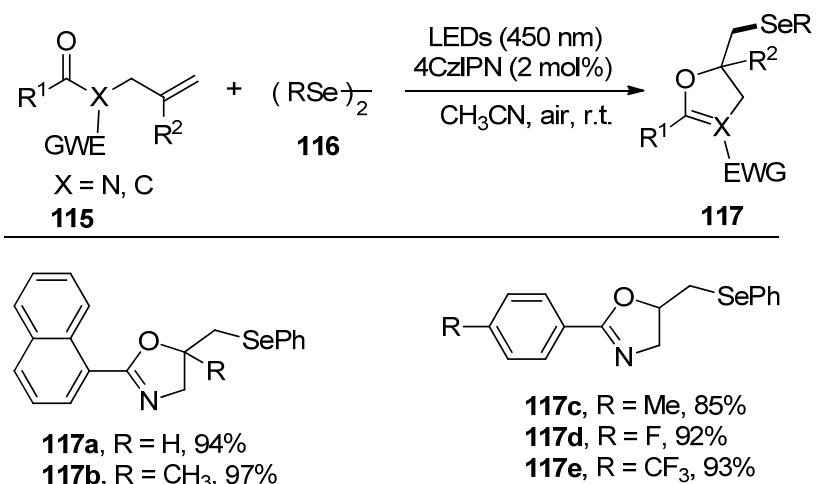

117b, $\mathrm{R}=\mathrm{CH}_{3}, 97 \%$
117a,

$117 \mathrm{e}, \mathrm{R}=\mathrm{CF}_{3}, 93 \%$

图式 34 光催化烯烃与二硒醚的脱氢环化

Scheme 34 Photocatalytic dehydrogenation and cyclization of olefins and selenium compounds

基于上述结果和文献报道 ${ }^{[47]}$, 提出了如 Scheme 35
所示的可能机理, 在可见光照射下, $4 \mathrm{CzlPN}$ 被转化为激 发态的 $4 \mathrm{CzlPN}^{*}$ 。二苯基二硒醚 17 与 $4 \mathrm{CzlPN} *$ 之间进行 单电子转移得到 $17^{\circ+}$ 自由基阳离子, 并且生成 $4 \mathrm{CzlPN}^{--}$ 自由基阴离子. 光氧化还原循环是通过分子氧氧化 $4 \mathrm{CzlPN}^{-}$到基态来完成的. 二硒自由基阳离子 $17^{\circ+}$ 随后 与 $N$-烯酰胺 115 反应生成硒态阳离子 118 和二苯二硒醚. 最后，通过中间体 118 的分子内亲核环化生成目标产物.

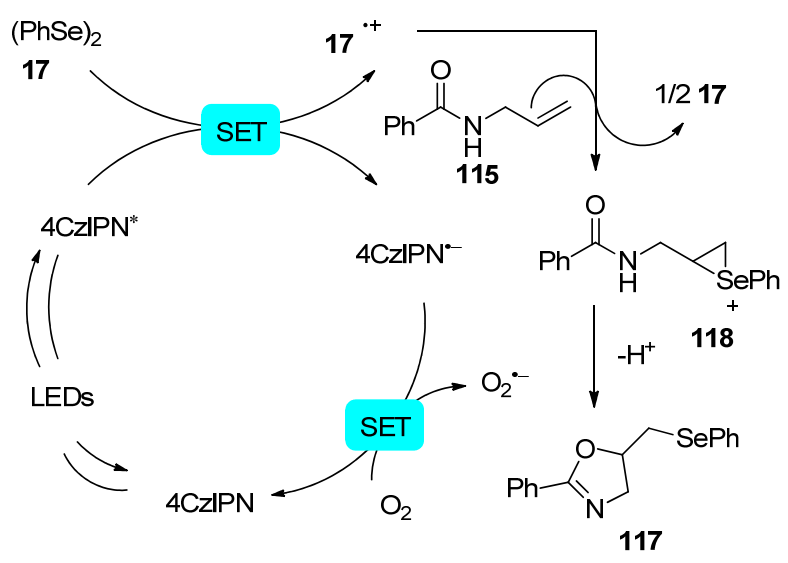

图式 35 可能的机理

Scheme 35 Possible mechanism

\section{3 电化学驱动的硒环化}

近年来，电化学在有机合成中被广泛认为是一种可 持续且环境友好的合成方法 ${ }^{[48]}$ 。电化学提供了一种超 越了经典的化学工艺的直接生成活性中间体的方法.

鉴于含硒化合物的重要性, 2019 年, 雷爱文课题 组 ${ }^{[49]}$ 报道了在没有金属催化剂和外加氧化剂的情况下, 烯烃羰基与二硒醚的电化学氧化环化反应, 为硒功能化 二氢呋喃的制备提供了一条既实用又经济的途径 (Scheme 36). 苯环对位/间位含有吸电子基团或给电子 基团的底物 119 均可以与二苯基二硒醚 17 顺利反应，以 中等至较好的产率得到相应的二氢呋喃化合物.

令人满意的是, 使用不饱和酰胺和 1,2-二苯基二硒 醚也成功地合成了一系列噁唑啉(Scheme 37). 其中, 含 富电子芳基的 $N$-烯丙基酰胺可以很容易地与二硒环构 筑 $\mathrm{C}-\mathrm{Se}$ 和 $\mathrm{C}-\mathrm{O}$ 键, 以中等到优异的产率得到相应的 硒代噁唑啉. 重要的是, 克级实验的成功和易于后续的 衍生化证明了该方案在制药和合成研究中的应用潜力.

为了探讨反应机理，在不同条件下进行了对照实 验. 为确定是否涉及自由基途径, 向体系中加入 TEMPO, 反应受到抑制. 除此之外, 还进行循环伏安实 验, 最终发现二苯基二硒醚(17)可能参与氧化和还原过 程. 根据上述实验结果和文献报道 ${ }^{[50]}$, 提出了如 Scheme 38 所示的反应机理. 首先, 二苯二硒醚在阴极 还原得到阴离子自由基中间体 123 , 进一步分解得到苯 

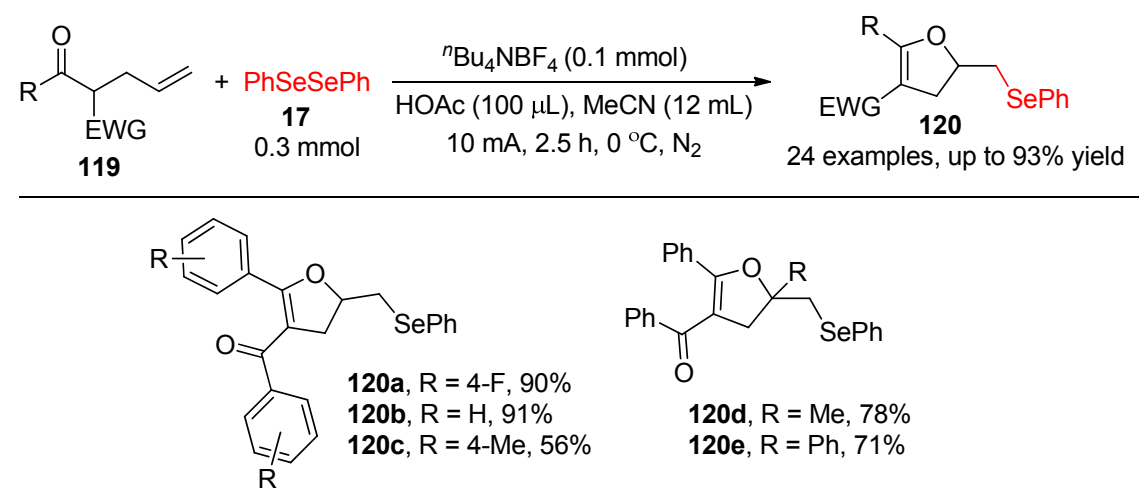

图式 36 电化学合成二氢呋喃

Scheme 36 Electrochemical synthesis of dihydrofuran
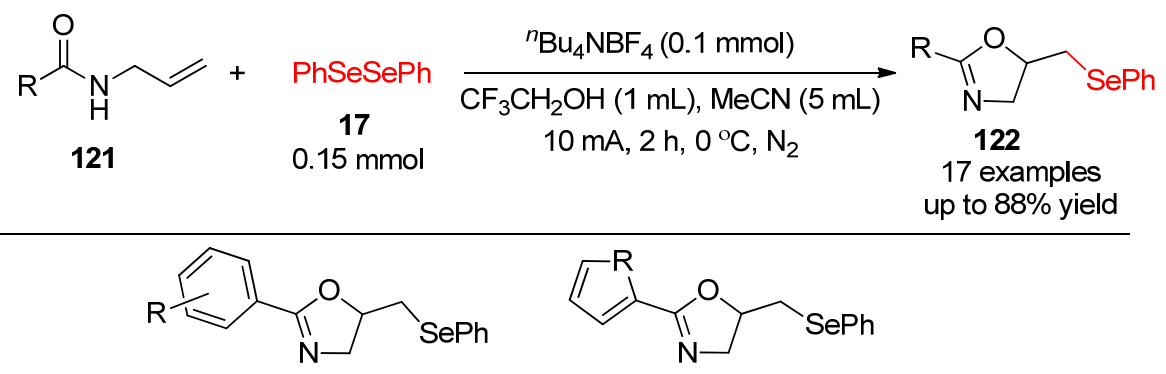

122a, $\mathrm{R}=\mathrm{H}, 88 \%$

122b, $\mathrm{R}=4-\mathrm{OMe}, 82 \%$

122d, $R=S, 72 \%$

122c, $R=4-F, 70 \%$

122e, $R=O, 68 \%$

图式 37 电化学合成噁唑啉

Scheme 37 Electrochemical synthesis of oxazoline

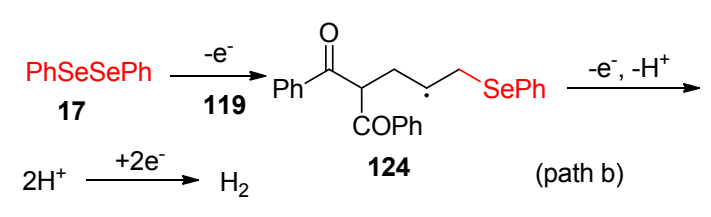<smiles>O=C(C1=C(c2ccccc2)OC(C[SeH]c2ccccc2)C1)c1ccccc1</smiles>

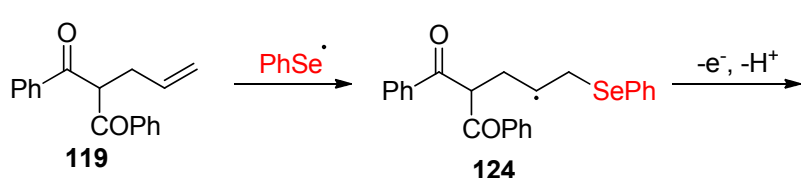

124

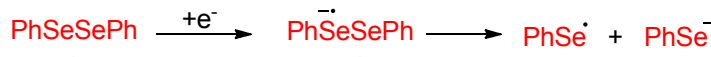

17

123

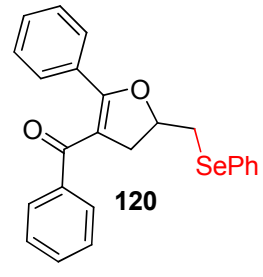

(path a)

图式 38 电化学合成二氢呋喃和噁唑啉的可能机理

Scheme 38 Possible mechanism electrochemical synthesis of dihydrofuran and oxazoline

基硒自由基和苯基硒阴离子. 然后, 苯基硒自由基被

119 的烯基捕获形成烷基自由基 124, 在羰基氧原子的 亲核攻击之后快速闭环, 最后通过去质子化过程提供所 需的产物(path a). 除此之外, 不能完全排除 119 与苯基 硒自由基反应生成烷基自由基 $\mathbf{1 2 4}$ (path b)的途径.

2020 年, De Sarkar 及其同事 ${ }^{[51]}$ 在电化学条件下, 以 酰胺类和肟类化合物为起始原料, 合成了硒化啞唑啉和
异噁唑啉衍生物(Scheme 39). 该反应只使用亚化学计 量的二硒醚, 反应在室温空气中下进行. 分别对几种底 物进行了扩展，反应对芳环的电子效应和空间效应均表 现出极好的耐受性. 此外，具有不同链长和不饱和度的 酰胺在氧化环化过程中也能较好地兼容. 这种不含外部 氧化剂和过渡金属的绿色方案反应时间短, 并且在大规 模合成中具有潜在的应用前景. 
<smiles>C=CCNC(=O)c1ccccc1</smiles>

125<smiles>[R]c1cccc(C(CC=CC(=O)O)=NO)c1</smiles>
127

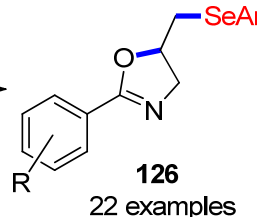

22 examples
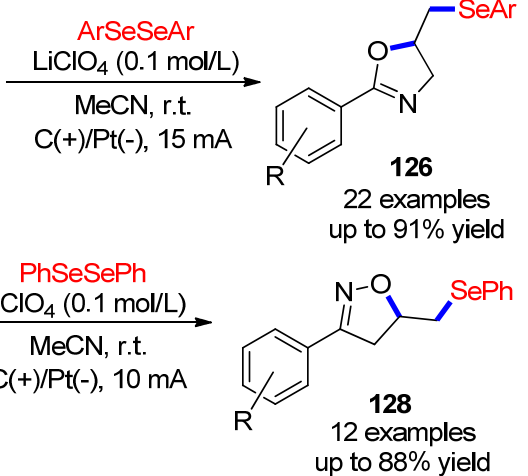

up to $88 \%$ yield
图式 39 电化学合成硒化噁唑啉和异噁唑啉衍生物 Scheme 39 Electrochemical synthesis of selenide oxazoline and isoxazoline derivatives

2019 年, 郭凯课题组 ${ }^{[52]}$ 报道了在无金属和无氧化 剂条件下, 电化学诱导烯醇酸酯或烷基酰胺与二硒醚的 氧化环化反应(Scheme 40). 底物 129 与芳基或烷基二硒 醚反应, 以较高的产率得到了相应的产物. 该方案具有 广泛的底物范围和良好的官能团兼容. 除此之外, 该反 应可以克级规模进行, 在未来的工业中具有实际应用价 值.

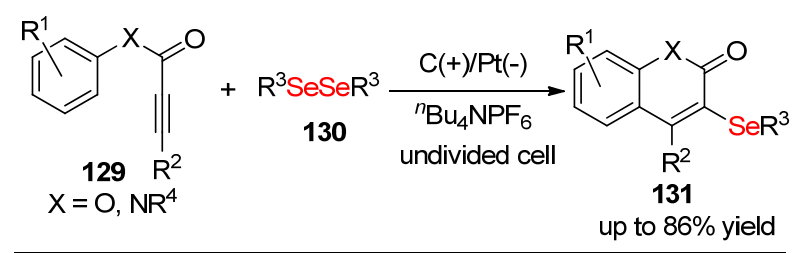<smiles>[R]c1ccc(-c2c(-c3ccccc3)c(=O)n(CC)c3ccc([R])cc23)cc1</smiles>

131a, $R^{1}=H, R^{2}=H, 85 \%$ 131b, $R^{1}=M e, R^{2}=H, 86 \%$ 131c, $R^{1}=\mathrm{Cl}, \mathrm{R}^{2}=\mathrm{H}, 81 \%$<smiles>[R]c1ccc(-c2c([Se]c3ccccc3)c(=O)oc3ccc([R])cc23)cc1</smiles>

131d, $R^{1}=M e, R^{2}=H, 85 \%$ 131e, $R^{1}=B r, R^{2}=H, 84 \%$
图式 40 电化学合成香豆素和喹啉酮类化合物

Scheme 40 Electrochemical synthesis of coumarin and quinolinone compounds

为了深入了解其机理，加入自由基捕获剂 TEMPO, 发现该电化学过程受到抑制, 证实该过程是通过自由基 途径进行的. 同时, 通过循环伏安实验测定了底物的氧 化还原电位, 结果表明二苯基二硒醚 17 在阳极上优先 氧化. 在上述结果和相关文献报道的基础上 ${ }^{[53]}$, 提出了 电化学氧化环化的可能机理(Scheme 41). 最初, 二苯基 二硒醚 17 在阳极氧化生成阳离子自由基中间体 132, 中 间体 132 再分解为苯硒基自由基 111 和苯硒阳离子 133 . 自由基 111 加到 129 的 $\mathrm{C} \equiv \mathrm{C}$ 键上, 生成了高区域选择
性的乙烯基自由基 134. 随后，乙烯基自由基 134 通过 分子内环化得到 135 , 再经过进一步的阳极氧化, 得到 中间体 136. 最后，中间体 136 通过去质子化和芳香族 体系的再生得到 3-硒化香豆素. 同时, 在下一次循环中, 苯硒阳离子 133 在阴极被还原为二苯基二硒醚.

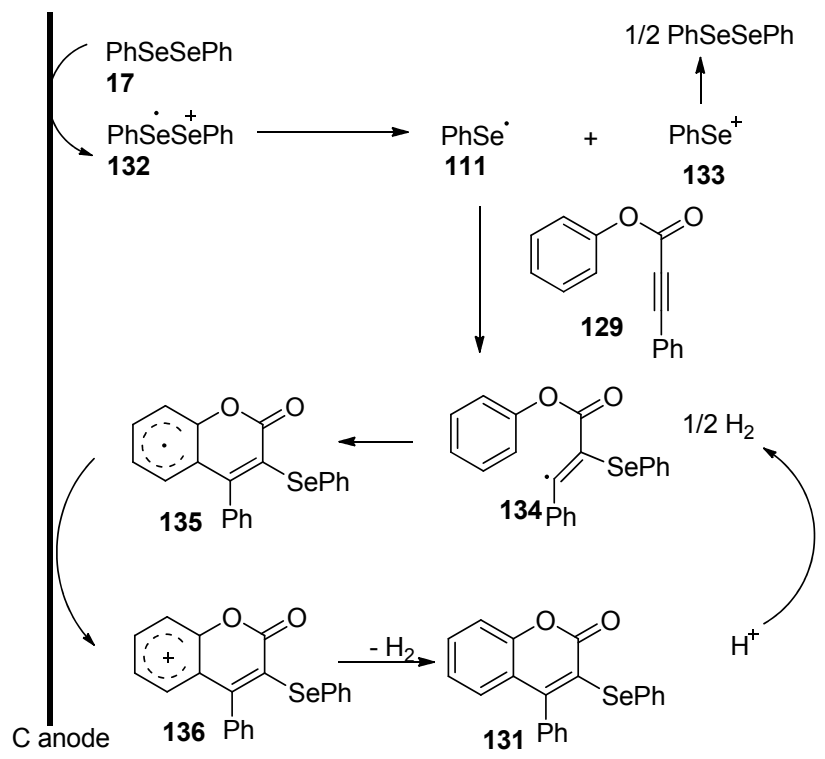

图式 41 电化学合成香豆素和喹啉酮类化合物的可能机理 Scheme 41 Possible mechanism electrochemical synthesis of coumarin and quinolinone compounds

在此方案的启发下, 2020 年, 该课题组 ${ }^{[54]}$ 进一步开 发了在电化学驱动下, 以炔烃与二硒醚为原料, 制备硒 螺[4.5]三烯酮的环化反应, 在 $n-\mathrm{Bu}_{4} \mathrm{NPF}_{6}$ 存在下, 恒流 为 $15 \mathrm{~mA}$ 的石墨阳极和铂阴极的整体电解槽中进行了 该反应(Scheme 42). 在最佳条件下，具有不同取代基的 底物 137 和 138 反应良好, 以中等至较好的产率获得了 预期的产物，表明反应具有较广的底物范围和较高的

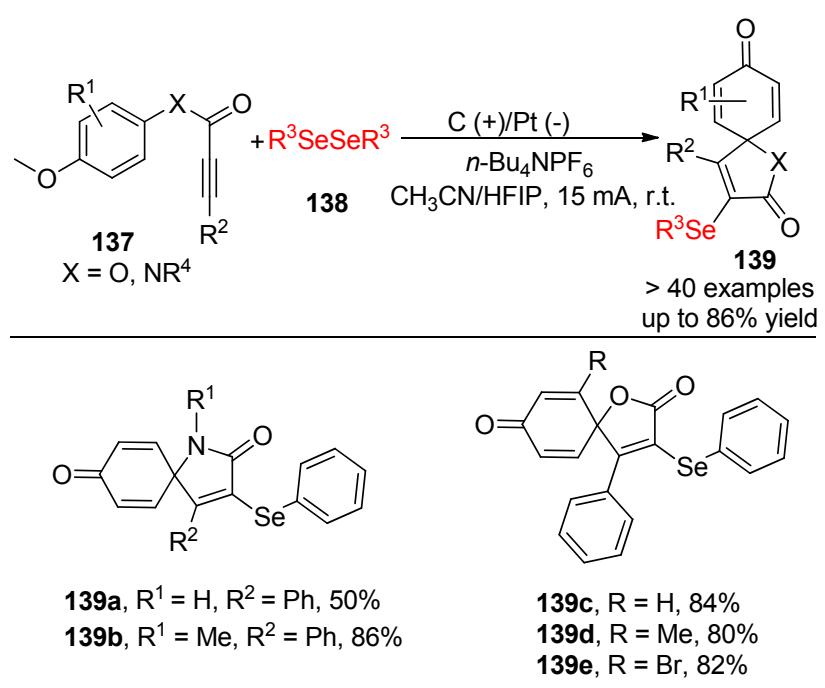

图式 42 电化学合成螺环 [4.5]三烯酮

Scheme 42 Electrochemical synthesis of spiro[4.5]trienones 
官能团耐受性. 值得注意的是, 端炔不适用于该体系, 除此之外, 借助电化学连续流动体系, 成功地进行了克 级反应, 证明了电化学与流动化学相结合的广阔前景.

将底物 137 在不存在二硒醚的条件下进行反应，但 没有检测到环化产物 139'。 反应结果表明螺环化合物 139'不是反应体系中的关键中间体，加入自由基捕获剂 TEMPO 和 BHT 后, 未检测到所需产物 139 (Scheme 43). 结果证实, 这一过程可能涉及自由基途径. 另外, 循环 伏安实验结果表明, 二苯二硒醚在阳极上优先氧化.

基于上述结果和相关文献报道 ${ }^{[55]}$, 提出了如 Scheme 43 所示的电化学转化的可能机理. 最初, 二苯 基二硒醚 17 被氧化, 形成阳离子自由基中间体 132. 然 后，中间体 132 转化苯硒基自由基 111 和苯硒阳离子 134. 此后, 111 和 137 通过自由基加成反应产生乙烯基 自由基 140. 140 通过分子内螺环反应生成中间体 141, 中间体 141 在阳极上进一步氧化, 得到含氧阳离子中间 体 142, 阳离子 142 脱甲基化和芳香环芳构化(path a)得 到产物 139. 除此之外, 不能排除中间体 143 由苯基硒 阳离子 134 和 137 提供的可能. 然后, 通过富电子芳环 对环状硒阳离子的分子内亲核攻击, 得到中间体 144 , 通过去甲基化得到产物 139 (path b).
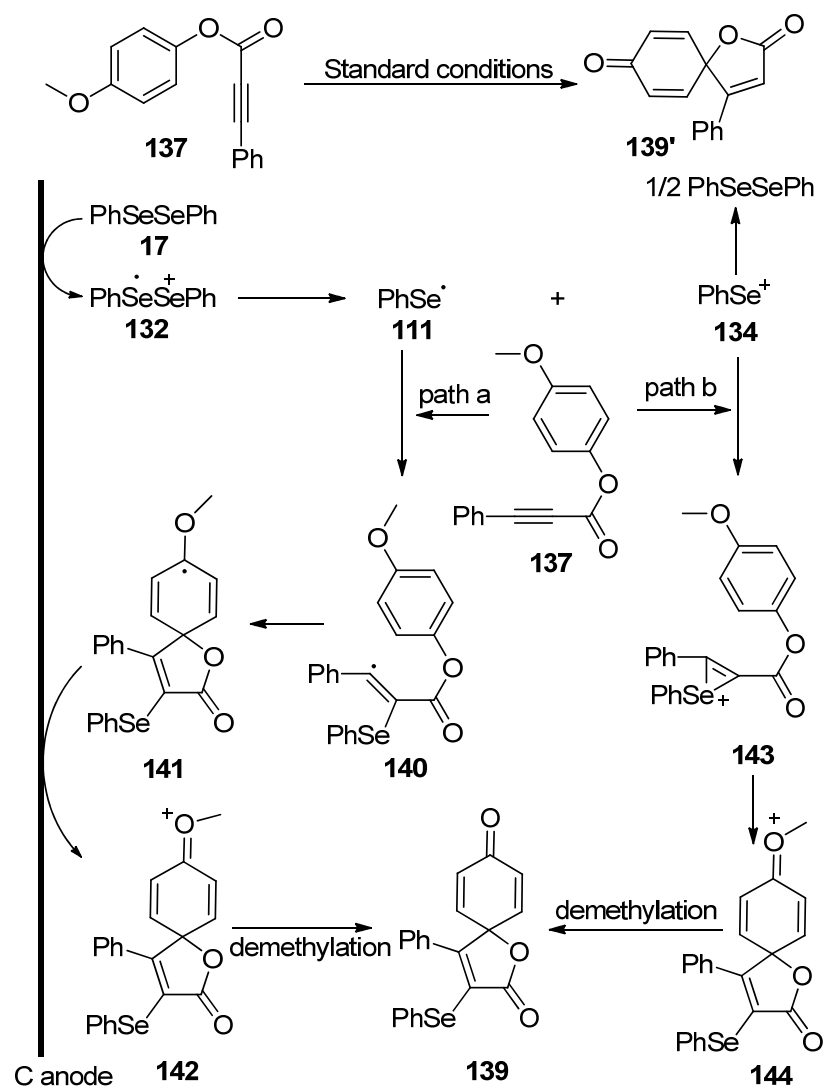

图式 43 螺 [4.5] 三烯酮的电化学合成机理

Scheme 43 Mechanism for electrochemical synthesis of spiro[4.5]trienones
2020 年, 郭维斯等 ${ }^{[56]}$ 开发了一种以 $N$-烯丙基硫代 酰胺为原料, 通过电化学硒化/环化的方法来构建含硒 噻唑类化合物的新策略，该策略是在室温空气中，无催 化剂和氧化剂的条件下进行的(Scheme 44). 令人满意 的是，带有给电子基和吸电子基的底物 145 和二硒醚反 应顺利，以中等到良好的产率得到了目标产物。该反应 具有底物范围广、操作简单及选择性好等特点.

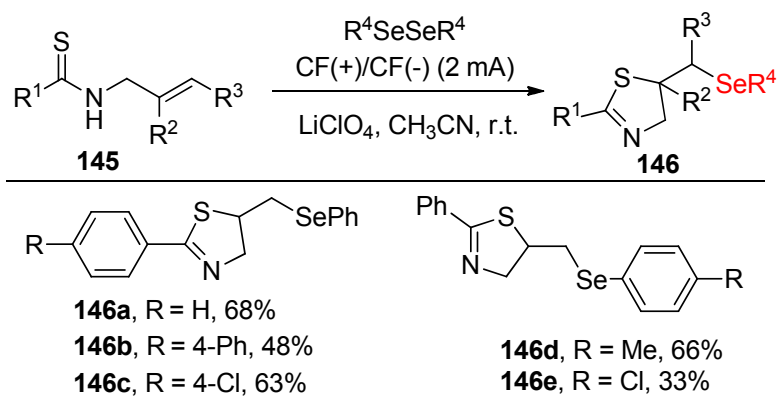

图式 44 电化学合成含硒噻唑啉

Scheme 44 Electrochemical synthesis of selenium-containing thiazoline

为了深入了解这一机制，进行了对照实验. 在自由 基捕获剂 TEMPO 存在下，仅检测到微量的产物，表明 可能存在自由基反应途径. 此外，还进行了自由基钟实 验，实验结果排除了分子内硫基自由基环化/硒化机理. 最后，进行了循环伏安实验，结果表明二硒醚的还原是 一个容易进行的途径.

基于对照实验，作者提出了如 Scheme 45 所示的可 能反应机理。首先，二硒醚 17 在阴极被还原成自由基 111 和硒阴离子 147. 中间体 147 很容易在阳极氧化生成 自由基 111. 随后，中间体 111 与 145 进行自由基加成生 成烷基自由基 148，148 在阳极上进一步氧化，得到碳

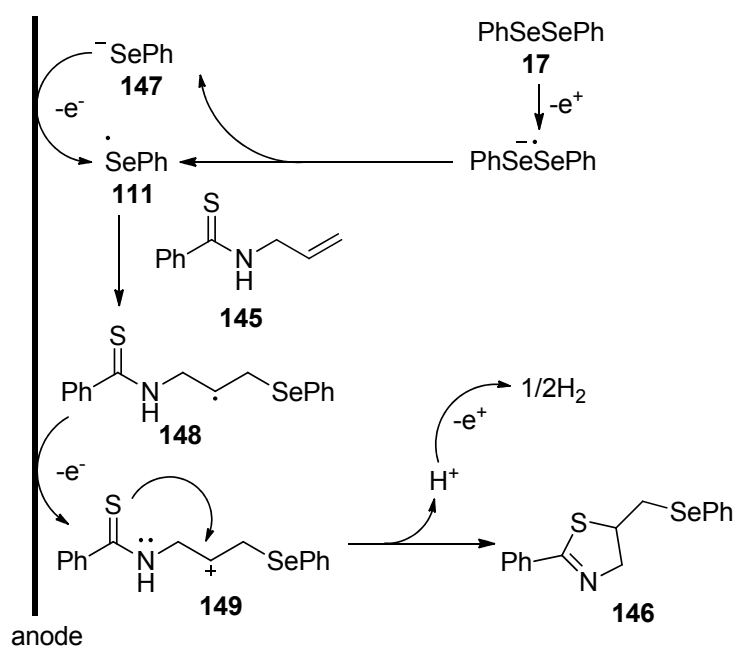

图式 45 噻唑啉的电化学合成机理

Scheme 45 Mechanism for the electrochemical synthesis of thiazoline 
阳离子 149. 最后, 149 进行区域选择性的分子内环化反 应/去质子化生成产物 146.

\section{4 硒参与其它类型的环化}

合成硒化苯并呋喃或苯并噻吩的开创性工作依赖 于使用商业上不可获得的、有毒且不稳定的 $\mathrm{RSeCl}$ 作为 硒源的亲电环化策略. 2002 年, Larock 课题组 ${ }^{[57]}$ 报道了 炔烃化合物与 $\mathrm{RSeCl}$ 的亲电环化反应, 以极高的产率合 成了 2,3-二取代苯并 $[b]$ 噻吩类化合物(Scheme 46).

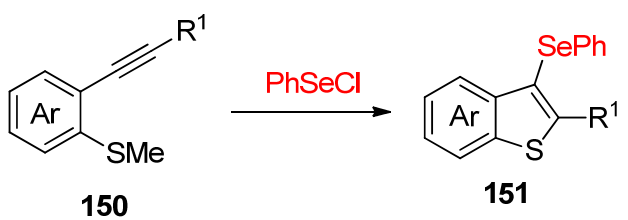

$\mathrm{R}^{1}=\mathrm{Ph}, n-\mathrm{C}_{8} \mathrm{H}_{17}, \mathrm{NC}\left(\mathrm{CH}_{2}\right)_{3}$, TMS, $t-\mathrm{Bu}$

图式 46 炔烃与硒的亲电环化

Scheme 46 Electrophilic cyclization of alkynes and selenium

2009 年, 黄宪及其同事 ${ }^{[58]}$ 以 $(E)-2$-(芳亚甲基)环丙 醛为原料, 通过不同有机硒试剂的介导的开环分子内自 由基环化反应和亲电环化反应, 分别合成 1-菜醛和 3-氧 杂双环 [3.1.0]己烷-2-醇(Scheme 47). 在优化的最佳条件 下，底物 152 芳环上取代基的性质和位置对反应影响不 大，产物产率较高.

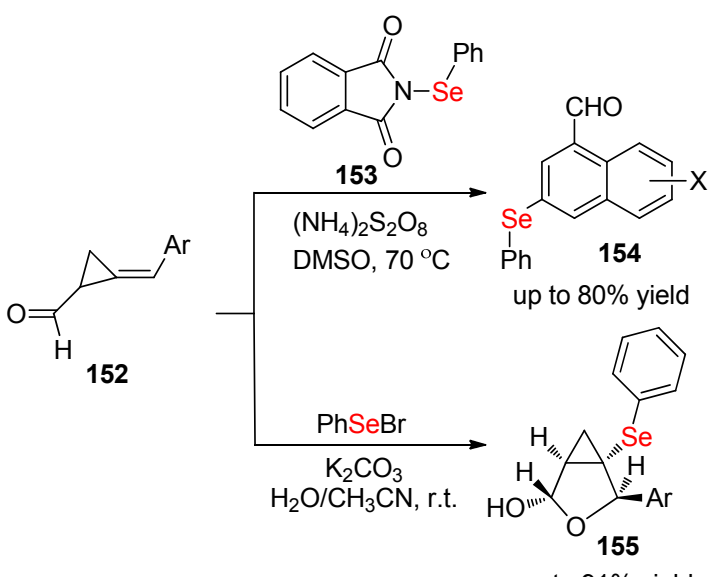

图式 47 环丙䤑的硒环化

Scheme 47 Selenium cyclization of cyclopropaldehyde

2012 年, Zeni 课题组 ${ }^{[59]}$ 以 1-丁基硒基-4-炔烃为原 料, 以碘为亲电源, 通过亲电环化反应合成了四氢硒苯 衍生物(Scheme 48), 含芳基的中性基团、富电子基团和 缺电子基团底物 156 都有效地得到环化产物. 该方案操 作简单, 反应条件温和, 以较高产率提供了环化的 5-exo-dig 产物.
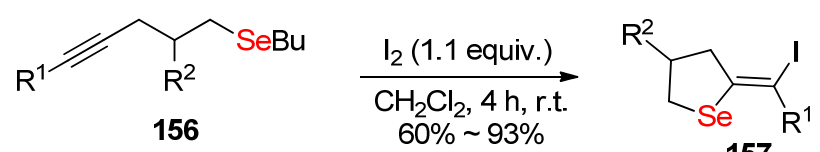

157

$\mathrm{R}^{1}=$ aryl, heteroaryl, alkyl, $\mathrm{SeBu}, \mathrm{Si}\left(\mathrm{CH}_{3}\right)_{3}$, propargyl alcohol $\mathrm{R}^{2}=\mathrm{H}, \mathrm{OH}, \mathrm{OBz}$

图式 48 炔烃化合物与碘的亲电环化

Scheme 48 Electrophilic cyclization of alkyne compounds with iodine

2012 年, 王䂞及其同事 ${ }^{[60]}$ 报道了在 $\mathrm{I}_{2}$ 催化的条件 下，2-(偕二溴乙烯基)- $N$-甲磺酰基苯胺与二苯二硒醚的 一锅反应，以良好的产率和较高的区域选择性得到了目 标产物(Scheme 49). 该方案为合成 2-溴-3-硒基吲哚提 供了一条新的、高效且简便的路线.

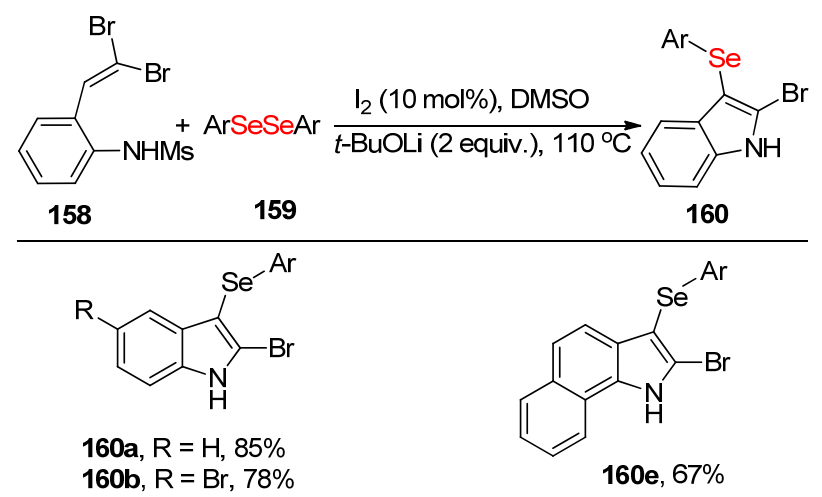

图式 49 碘催化甲磺酰基苯胺与二苯二硒醚的环化 Scheme 49 Iodine catalyzed cyclization of methanesulphonylaniline and diphenylselenide

硒烯是公认的一类在材料科学中表现出广泛生物 活性 ${ }^{[61]}$ 和应用潜力的有机硒化合物. 2015 年, Zeni 课题 组 ${ }^{[62]}$ 报道了以 $\left[2\right.$-(丁硒酰基)苯基]丙炔醇为底物, 在 $\mathrm{I}_{2}$ 催化条件下发生的亲核环化反应(Scheme 50), 一系列 底物反应良好，以较高的产率生成了多种 2-酰基硒苯类 化合物.

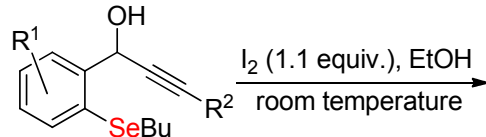

161

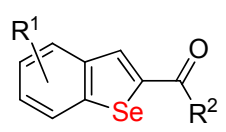

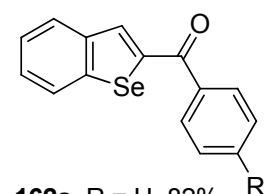

162a, $\mathrm{R}=\mathrm{H}, 82 \% \quad \mathrm{R}$

162b, $\mathrm{R}=\mathrm{Me}, 72 \%$

162c, $\mathrm{R}=\mathrm{Cl}, 69 \%$

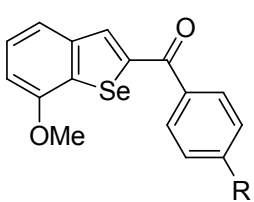

162d, $\mathrm{R}=\mathrm{OMe}, 65 \%$ 162e, $\mathrm{R}=\mathrm{Cl}, 45 \%$
图式 50 碘催化丙炔醇的环化

Scheme 50 Cyclization of propargyl alcohol catalyzed by iodine

2018 年, 董春娥及其同事 ${ }^{[63]}$ 以 1,4-二氮双环[2.2.2] 
辛烷(DABCO)为催化剂, 在温和的反应条件, 实现了烯 胺与苯硒酰氯的环化反应, 多数取代的苯甲酰胺 163 都 以较高的产率生成一系列异苯并呋喃-1(3H)-亚胺衍生 物(Scheme 51), 该方法将成为获得苯硒杂环化合物的 便捷途径.

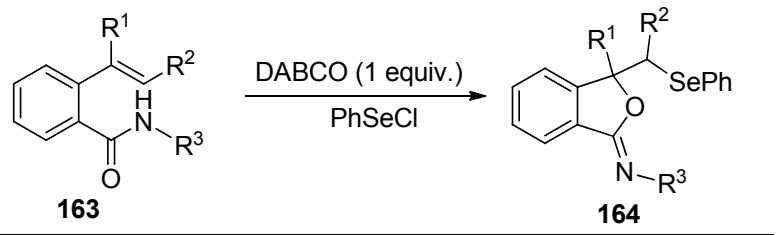<smiles>[R]N=C1OC(C)(C[Se]c2ccccc2)c2ccccc21</smiles>

164a, $\mathrm{R}=\mathrm{Me}, 91 \%$ 164b, $R=A d, 68 \%$

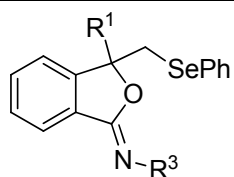

164c, $\mathrm{R}^{1}=4-\mathrm{Br}_{-} \mathrm{C}_{6} \mathrm{H}_{4}, \mathrm{R}^{3}=4-\mathrm{F}-\mathrm{C}_{6} \mathrm{H}_{4}, 74 \%$ 164d, $\mathrm{R}^{1}=4-\mathrm{Cl}-\mathrm{C}_{6} \mathrm{H}_{4}, \mathrm{R}^{3}=\mathrm{Ph}, 81 \%$

图式 51 DABCO 催化烯胺的环化

Scheme 51 Cyclization of enamine catalyzed by 1,4-diazabicyclo[2.2.2] octane

2018 年, 王海课题组 ${ }^{[64]}$ 报道了以 ${ }^{t} \mathrm{BuOK}$ 为启动子、 以 TFBen 为 $\mathrm{CO}$ 源, 在温和的条件下, 炔丙基胺和硒元 素进行环化反应合成 1,3-硒唑烷-2-酮. 这是一种新的、 简单的羰基化反应过程(Scheme 52). 其中, 富电子或缺 电子基团取代的炔丙基胺均以较高产率生成相应的产 物, 而带有 $N$-环已基单元的炔丙胺没有得到预期的产 物, 这可能受氮原子的立体结构的影响。

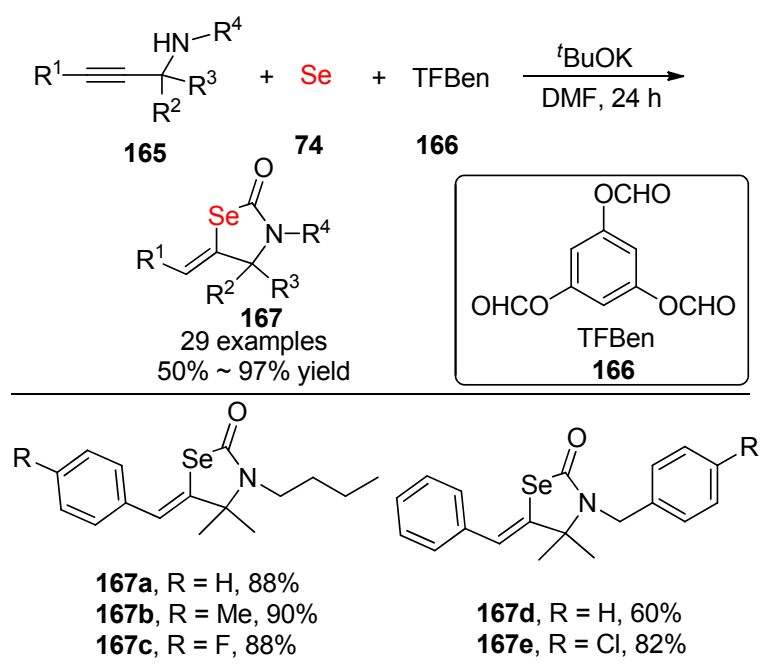

图式 52 炔烃化合物与硒的环化

Scheme 52 Cyclization of alkyne compounds with selenium

2019年, 刘雪原及其同事 ${ }^{[65]}$ 报道了由 TBHP 引发的 炔丙基芳基醚与二芳基二硒醚的自由基环化反应, 合成 了多种 3-有机硒基- $2 H$-香豆素(Scheme 53). $N$-碘代丁二
酰亚胺(NIS)可以与二芳基二硒醚原位生成 ArSeI，提高 反应产率. 该反应具有良好的官能团兼容性与广泛的底 物范围，以中等至优异的产率同时构筑 $\mathrm{C}-\mathrm{Se}$ 键、 $\mathrm{C}-\mathrm{C}$ 键和 $\mathrm{C}=\mathrm{O}$ 键, 这种转化在药物和生物活性化合物的合 成中具有潜在的应用前景.
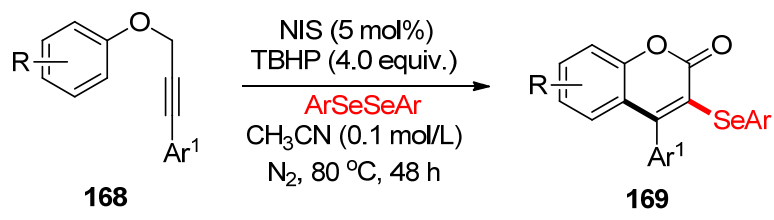

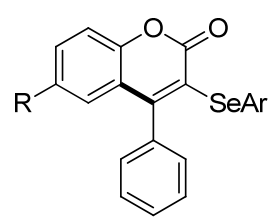

$169 a, R=M e, 82 \%$ 169b, $\mathrm{R}=\mathrm{Ph}, 91 \%$ 169c, $\mathrm{R}=\mathrm{Cl}, 80 \%$

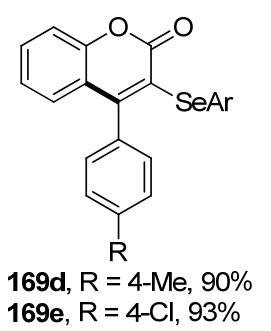

$169 e, R=4-\mathrm{Cl}, 93 \%$
图式 53 炔丙基芳基醚与二芳基二硒醚环化

Scheme 53 Cyclization of propargyl aryl ether and diaryl diselenide

为了进一步了解反应机理, 在标准反应体系中加入 TEMPO、BHT 或 1,1-二苯乙烯，并通过气相色谱一质谱 联用技术(GC-MS)检测到自由基捕获产物。除此之外, 在没有 NIS 的情况下, $\mathrm{O}_{2}$ 可以抑制该反应，结果表明该 环化过程可能涉及自由基途径.

基于上述结果和之前的报道 ${ }^{[66-67]}$, 作者提出了合理 的机理(Scheme 54). 值得注意的是, 含硒化合物 171 可 能是关键中间体. $\mathrm{PhSe}$-自由基在中间体 $\mathbf{1 7 1}$ 的参键上区 域选择性地环化生成中间体 175. 否则, PhSeI 与中间体 171 的亲电环化也是可能的. 随后, 从中间体 175 生成 169 的氧化方法可以通过 Pummerer 型反应得出, 其中溶 剂中的 $\mathrm{H}_{2} \mathrm{O}$ 是亲核试剂, 产生醇 $\mathbf{1 7 5}^{\prime}$. 接着在高浓度的 TBHP 作用下, 进一步氧化该醇生成羰基结构. 除了这 条路线外，还有另一条通往所需产物的可能途径，中间 体 175 通过叔丁氧基的烯丙基抽氢生成自由基中间体 176, 它很容易被叔丁基过氧自由基捕获，生成中间体 $177^{[68]}$, 然后中间体 177 同时裂解 $\mathrm{C}-\mathrm{Se}$ 键和 $\mathrm{O}-\mathrm{O}$ 键, 生成所需产物。

2019 年, Baidya 课题组 ${ }^{[69]}$ 以廉价的 $\mathrm{K}_{2} \mathrm{~S}_{2} \mathrm{O}_{8}$ 为氧化 剂，在无金属条件下实现了 $N$-芳基烷基酰胺的级联环 化反应，合成了 3-硒基喹啉-2-酮和 3-硒螺 [4.5] 三烯酮 (Scheme 55). 该反应对带有不同给电子和吸电子基团 的底物 178 均表现出极好的耐受性，产率高达 $98 \%$. 除 此之外，当使用 $N$-芳环上具有邻甲氧基取代的底物时， 以良好分离收率获得了具有挑战性的含邻醌骨架的螺 环产物. 


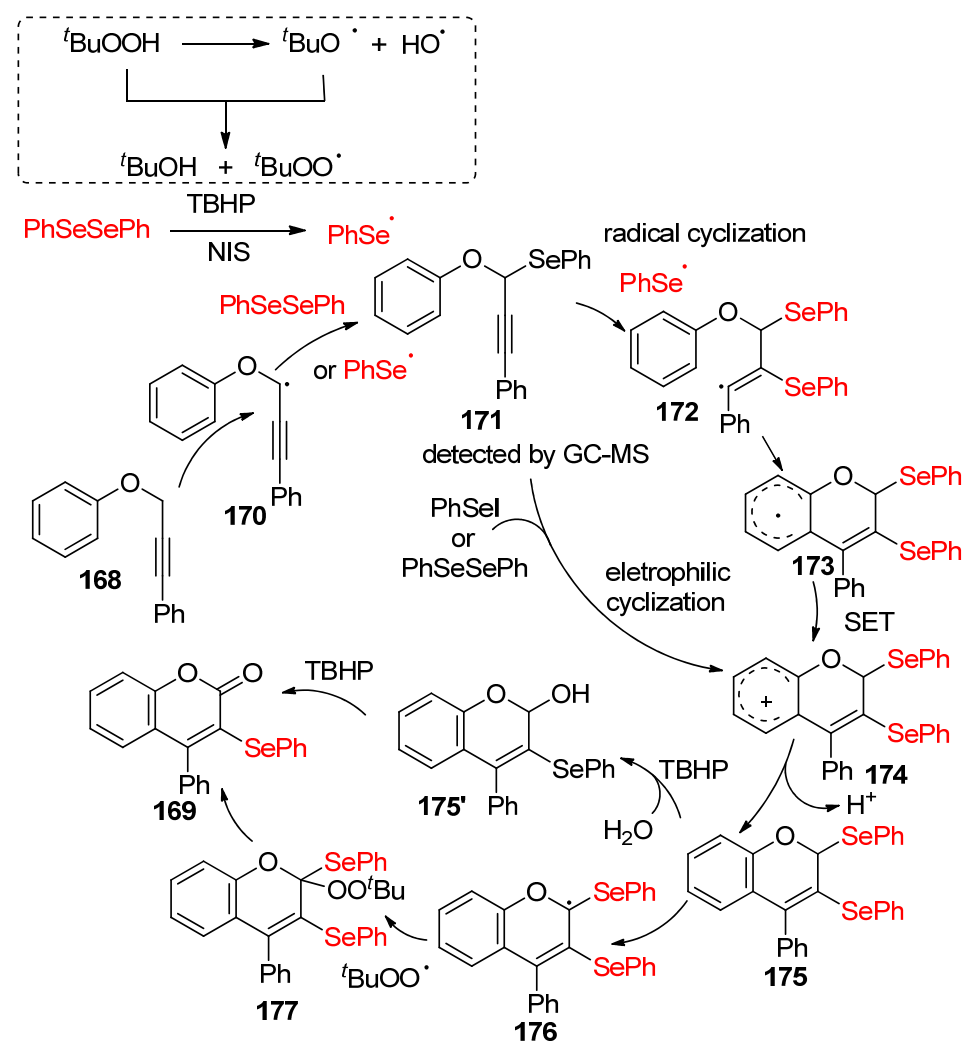

图式 54 炔丙基芳基醚与二芳基二硒醚环化可能的机理

Scheme 54 Proposed mechanism cyclization of propargyl aryl ether and diaryl diselenide

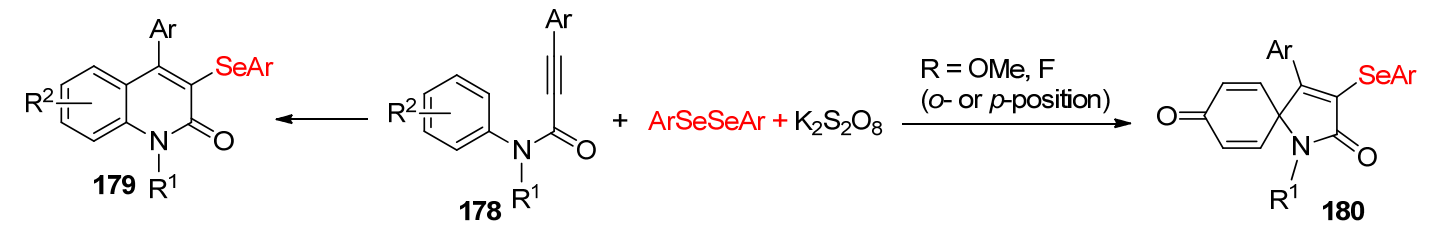<smiles>[R]c1ccc([Se]c2c(-c3ccccc3)c3ccccc3n(C)c2=O)cc1</smiles>

179a, $\mathrm{R}=\mathrm{Cl}, 95 \%$ 179b, $R=M e, 98 \%$<smiles>[R]c1ccc([Se]C2=C(Br)C3(C=CC(=O)C=C3)N(C)C2=O)cc1</smiles>

180a, $\mathrm{R}=\mathrm{Me}, 73 \%$ 180b, $\mathrm{R}=\mathrm{Cl}, 74 \%$<smiles>CN1C(=O)C(Br)=C(Br)C1=O</smiles>

$180 c, 52 \%$

图式 55 环化反应合成三烯酮

Scheme 55 Synthesis of triketene by cyclization

作者提出了自由基途径的假设, 并得到了对照实验 结果的支持 (Scheme 56). 在该可行途径中, 在 $\mathrm{K}_{2} \mathrm{~S}_{2} \mathrm{O}_{8}$ 作 用下, 由 $\mathrm{ArSeSeAr}$ 生成的芳基硒自由基与 $N$-芳基烷基 酰亚胺 178 反应生成自由基中间体 181, 它经过分子内 螺环反应来合成中间体 182,182 进一步氧化得到中间体 183, 中间体 183 经过扩环生成中间体 184, 其在芳构化 的基础上生成喹诺酮产物. 在 $N$-芳基烷基酰亚胺含有 对位 F/OMe 取代基的情况下, 中间体 182 进一步与溶剂 化分子氧反应生成中间体 185 . 然后, 中间体 185 经历 $\mathrm{O}-\mathrm{O}$ 键断裂, 进行脱氟/去甲氧基化, 得到所需的产物.

\section{5 结论与展望}

对近年来有机硒参与的环化新方法，按照金属催 化、电化学驱动、可见光驱动及有机分子催化等类型进 行了较为详细地介绍, 并对反应的部分机理进行了讨 论. 从上述讨论可以看出, 将有机硒引入杂环化合物中 进行衍生化修饰已经引起合成化学家的广泛关注, 并且 已经取得了很大的进步. 有机硒参与的环化反应, 为呋 喃、吲哚、噁唑及喹啉等杂环化合物的合成提供了经济、 高效的合成途径. 

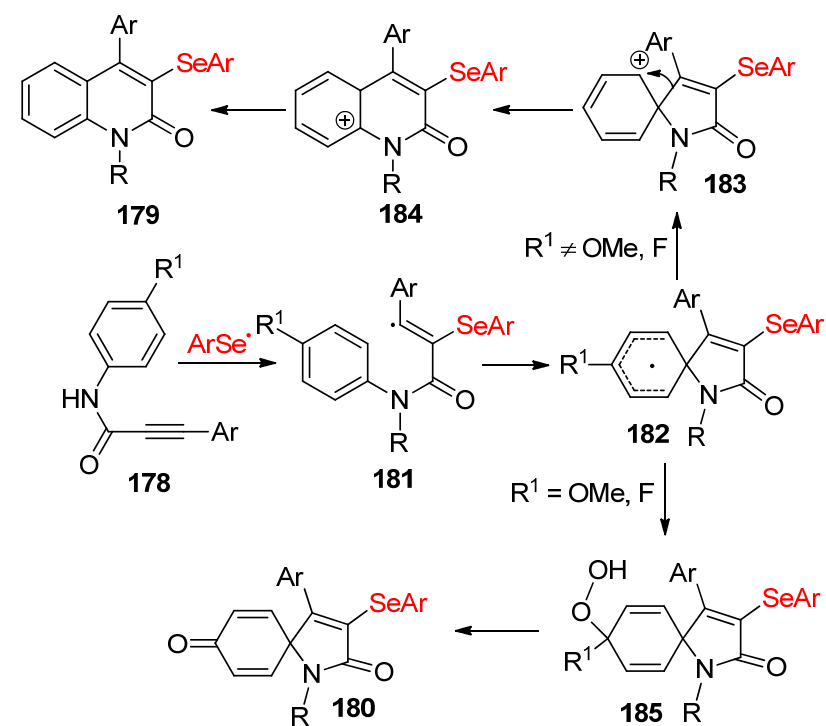

图式 56 合成三烯酮的可能机理

Scheme 56 Possible mechanism of synthesis of triketene

尽管此类反应已取得重大进展, 但多数工作需在高 温或过渡金属催化剂存在条件下进行. 电化学驱动和可 见光驱动的有机硒参与的环化反应虽有报道但普适性 不够广泛. 因此, 在发展传统反应的同时, 通过引入可 见光或电化学等其他形式的催化体系, 开发新型高效、

经济实用的有机硒参与的硒环化反应, 并将其应用于工 业化生产与药物研发, 为后期值得关注的发展方向, 其 反应机理值得深入研究.

\section{References}

[1] (a) Zeni, G.; Braga, A. L.; Stefani, H. A. Acc. Chem. Res. 2003, 36, 731.

(b) Peng, X.-L.; Ma, C.; Tung, C.; Xu, Z.-H. Org. Lett. 2016, 18, 4154 .

(c) Huang, S.; Li, H.; Xie, T.; Wei, F.; Tung, C.; Xu, Z.-H. Org. Chem. Front. 2019, 6, 1663.

(d) Wang, W.-G.; Huang, S.; Yan, S.-K.; Sun, X.-J.; Tung, C.; Xu, Z.-H. Chin. J. Chem. 2020, 38, 445.

[2] Some examples: (a) Vinogradova, E. V.; Zhang, C.; Spokoyny, A. M.; Pentelute, B. L.; Buchwald, S. L. Nature 2015, 526, 687.

(b) Nogueira, C. W.; Zeni, G.; Rocha, J. B. T. Chem. Rev. 2004, 104, 6255 .

(c) Manna, D.; Roy, G.; Mugesh, G. Acc. Chem. Res. 2013, 46, 2706.

(d) Reich, H. J.; Hondal, R. J. ACS Chem. Biol. 2016, 11, 821.

[3] Guan, Q.; Han, C.-M.; Zuo, D.-Y.; Zhai, M.-A.; Li, Z.-Q.; Zhang, Q.; Zhai, Y.-P.; Jiang, X.-W.; Bao, K.; Wu, Y.-L.; Zhang, W.-G. Eur. J. Med. Chem. 2014, 87, 306.

[4] Sajomsang, W.; Tantayanon, S.; Tangpasuthadol, V.; Daly, W. H. Carbohydr. Res. 2009, 344, 2502.

[5] Wen, Y.; Xu, J.-W.; Wang, Z.-W.; Qi, H.; Xu, Q.-L.; Bai, Z.-S.; Zhang, Q.; Bao, K.; Wu, Y.-L.; Zhang, W. G. Eur. J. Med. Chem. 2015, 90, 184.

[6] Kumar, S.; Sharma, N.; Maurya, I. K.; Bhasin, A. K. K.; Wangoo, N.; Brandao, P.; Fleix, V.; Bhasin, K. K.; Kumar, R. K. Eur. J. Med. Chem. 2016, 123, 916.

[7] (a) Brutchey, R. L. Acc. Chem. Res. 2015, 48, 2918.

(b) Patra, A.; Wijsboom, Y. H.; Leitus, G.; Bendikov, M. Chem.
Mater. 2011, 23, 896.

[8] (a) Nogueira, C. W.; Rocha, J. B. T. Arch. Toxicol. 2011, 85, 1313. (b) Nogueira, C. W.; Zeni, G.; Rocha, J. B. T. Chem. Rev. 2004, 104, 6255 .

[9] Some examples: (a) Nunes, V. L.; de Oliveira, I. C.; Barros, O. S. D. Eur. J. Org. Chem. 2014, 2014, 1525.

(b) Santi, C.; Santoro, S.; Testaferri, L.; Tiecco, M. Synlett 2008, 1471 .

(c) Santi, C.; Santoro, S. In Organoselenium Chemistry: Synthesis and Reactions, Ed.: Wirth, T., Wiley-VCH, Germany, 2011, pp. 1 51.

(d) Ogawa, A.; Ogawa, I.; Obayashi, R.; Umezu, K.; Doi, M.; Hirao, T. J. Org. Chem. 1999, 64, 86.

[10] Some examples: (a) Perin, G.; Lenardão, E. J.; Jacob, R. G.; Panatieri, R. B. Chem. Rev. 2009, 109, 1277.

(b) Sun, K.; Shi, Z.; Liu, Z.; Luan, B.; Zhu, J.; Xue, Y. Org. Lett. 2018, 20, 6687.

(c) Sun, K.; Wang, X.; Lv, Y.; Li, G.; Jiao, H.; Dai, C.; Li, Y.; Zhang, C.; Liu, L. Chem. Commun. 2016, 52, 8471.

(d) Sun, K.; Wang, X.; Fu, F.; Zhang, C.; Chen, Y.; Liu, L. Green Chem. 2017, 19, 1490.

(e) Wang, X.; Mu, S.-Q.; Sun, T.; Sun, K. Chin. J. Org. Chem. 2019, 39, 2802 (in Chinese).

(王薪, 穆石强, 孙婷, 孙凯, 有机化学, 2019, 39, 2802.)

(f) Sun, K.; Wang, X.; Li, C.; Wang, H.; Li, L. Org. Chem. Front. 2020, 7,3100 .

[11] Some examples: (a) Bax, B. D.; Chan, P. F.; Eggleston, D. S.; Fosberry, A.; Gentry, D. R.; Gorrec, F.; Giordano, I.; Hann, M. M.; Hennessy, A.; Hibbs, M.; Huang, J.; Jones, E.; Jones, J.; Brown, K. K.; Lewis, C. J.; May, E. W.; Saunders, M. R.; Singh, O.; Spitzfaden, C. E.; Shen, C.; Shillings, A.; Theobald, A. J.; Wohlkonig, A.; Pearson, N. D.; Gwynn, M. N. Nature 2010, 466, 935.

(b) Rouffet, M.; de Oliveira, C. A. F.; Udi, Y.; Agrawal, A.; Sagi, I.; McCammon, J. A.; Cohen, S. M. J. Am. Chem. Soc. 2010, 132, 8232 .

(c) Andrews, S.; Burgess, S. J.; Skaalrud, D.; Kelly, J. X.; Peyton, D. H. J. Med. Chem. 2010, 53, 916.

(d) Sun, K.; Li, Y.; Feng, R.; Mu, S.; Wang, X.; Zhang, B. J. Org. Chem. 2020, 85, 1001.

(e) Sun, K.; Li, G.; Li, Y.; Yu, J.; Zhao, Q.; Zhang, Z.; Zhang, G. Adv. Synth. Catal. 2020, 362, 1947.

(f) Wang, X.; Li, G.; Sun, K.; Zhang, B. Chin. J. Org. Chem. 2020, 40,913 (in Chinese).

(王薪, 李国锋, 孙凯, 张冰, 有机化学, 2020, 40,913.)

(g) Sun, K.; Wang, X.; Wang, Q.; Xue, Y.; Wu, L.; Zhang, B. Chem. Commun. 2020, 56, 4436.

(h) Meng, X.; Kang, Q.; Zhang, J.; Li, Q.; Wei, W.; He, W. Green Chem. 2020, 22, 1388.

(i) Kang, Q.; Wu, W.; Li, Q.; Wei, W. Green Chem. 2020, 22, 3060.

[12] Some examples: (a) Cherney, A. H.; Kadunce, N. T.; Reisman, S. E. Chem. Rev. 2015, 115, 9587.

(b) Finkbeiner, P.; Kloeckner, U.; Nachtsheim, B. J. Angew. Chem., Int. Ed. 2015, 54, 4949.

(c) Riedmuller, S.; Kaulfhold, O.; Spreitzer, H.; Nachtsheim, B. J. Eur. J. Org. Chem. 2014, 2014, 1391.

(d) Liu, C.; Zhang, H.; Shi, W.; Lei, A. Chem. Rev. 2011, 111, 1780.

[13] Some examples: (a) Beletskaya, I. P.; Sigeev, A. S.; Peregudov, A. S.; Petrovskii, P. V. J. Organomet. Chem. 2000, 605, 96.

(b) Beletskaya, I. P.; Ananikov, V. P. Pure Appl. Chem. 2007, 79, 1041.

[14] Stein, A. L.; Alves, D.; da Rocha, J. T.; Nogueira, C. W.; Zeni, G. Org. Lett. 2008, 10, 4983.

[15] Du, H.-A.; Zhang, X.-G.; Tang, R.-Y.; Li, J.-H. J. Org. Chem. 2009, 74,7844 .

[16] Gay, R. M.; Manarin, F.; Schneider, C. C.; Barancelli, D. A.; Costa, M. D.; Zeni, G. J. Org. Chem. 2010, 75, 5701.

[17] Li, Z.; Hong, L.; Liu, R.; Shen, J.; Zhou, X. Tetrahedron 2011, 52, 1343. 
[18] Schumacher, R. F.; Rosario, A. R.; Leite, M. R.; Zeni, G. ChemInform 2013, 19, 13059.

[19] Mantovani, A. C.; Goulart, T. A. C.; Back, D. F.; Menezes, P. H.; Zeni, G. J. Org. Chem. 2014, 79, 10526.

[20] Maity, P.; Kundu, D.; Roy, R.; Ranu, B. C. Org. Lett. 2014, 16, 4122 .

[21] Reddy, A. S.; Swamy, K. C. Org. Lett. 2015, 17, 2996.

[22] Stein, A. L.; Rosario, A. R.; Zeni, G. Eur. J. Org. Chem. 2015, 2015,5640 .

[23] Wang, W.; Peng, X.; Wei, F.; Tung, C.; Xu, Z. Angew. Chem., Int. Ed. 2016, 55, 649 .

[24] Yamada, M.; Matsumura, M.; Takino, F.; Murata, Y.; Kurata, Y.; Kawahata, M.; Yamaguchi, K.; Kakusawa, N.; Yasuike, S. Eur. J. Org. Chem. 2018, 2018, 170.

[25] Some examples: (a) Yamada, M.; Matsumura, M.; Uchida, Y.; Kawahata, M.; Murata, Y.; Kaku-sawa, N.; Yamaguchi, K.; Yasuike, S. Beilstein J. Org. Chem. 2016, 12, 13093.

(b) Yamada, M.; Matsumura, M.; Murata, Y.; Kawahata, M.; Saito, K.; Kakusawa, N.; Yamaguchi, K.; Yasuike, S. Tetrahedron 2017, 73,2614 .

[26] Some examples: (a) Ricordi, V. G.; Thurow, S.; Penteado, F.; Schumacher, R. F.; Perin, G.; Lenardão, E. J.; Alves, D. Adv. Synth. Catal. 2015, 357, 933.

(b) Stein, A. L.; Bilheri, F. N.; Rocha, J. T.; Back, D. F.; Zeni, G. Chem.-Eur. J. 2012, 18, 10602.

(c) Beletskaya, I. P.; Sigeev, A. S.; Peregudov, A. S.; Petrovskii, P. V.; Khrustalev, V. N. Chem. Lett. 2010, 39, 720.

[27] Goulart, T. A. C.; Back, D. F.; Zeni. G. Adv. Synth. Catal. 2017, 359, 1901.

[28] Kaz-mierczak, J. C.; Recchi, A. M. S.; Gritzenco, F.; Balbom, E. B.; Barcellos, T.; Sperança, A.; Godoi, B. Eur. J. Org. Chem. 2017, 2017, 6382.

[29] Casola, K. K.; Gomes, M. R.; Back, D. F.; Zeni, G. J. Org. Chem. 2018, 83,6706

[30] Cui, F.; Chen, J.; Mo, Z.; Su, S.; Chen, Y.; Ma, X.; Tang, H.; Wang, H.; Pan, Y.; Xu, Y.-L. Org. Lett. 2018, 20, 925.

[31] An, C.; Li, C.-Y.; Huang, X.-B.; Gao, W.-X.; Zhou, Y.-B.; Liu, M.-C.; Wu, H.-Y. Org. Lett. 2019, 21, 6710.

[32] Some examples: (a) Yan, J.; Xu, J.; Zhou, Y.; Chen, J.; Song, Q. Org. Chem. Front. 2018, 5, 1483.

(b) Xu, J.; Yu, X.; Yan, J.; Song, Q. Org. Lett. 2017, 19, 6292.

(c) Liu, W.; Hu, Y.-Q.; Hong, X.-Y.; Li, G.-X.; Huang, X.-B.; Gao, W.-X.; Liu, M.-C.; Xia, Y.-Z.; Zhou, Y.-B.; Wu, H.-Y. Chem. Commun. 2018, 54, 14148 .

[33] Sun, K.; Wang, S.; Feng, R.; Zhang, Y.; Wang, X.; Zhang, Z.; Zhang, B. Org. Lett. 2019, 21, 2052.

[34] Ren, P.-X.; Qi, L.; Fang, Z.-Y.; Wu, T.-S.; Gao, Y.-M.; Shen, S.; Song, J.-Y.; Wang, L.-J.; Li, W. Chin. J. Org. Chem. 2019, 39, 1776 (in Chinese).

(任培星, 齐林, 方卓越, 吴天舒, 高雅蒙, 沈松, 宋金燕, 王力 竞, 李玮，有机化学, 2019, 39, 1776.)

[35] Zhou, X.-G.; Wang, S.-K.; Li, Z.; Liu, R.-T. Chin. J. Org. Chem. 2019, 39, 3215 (in Chinese). (周锡庚, 王圣克, 李振, 刘瑞婷, 有机化学, 2019, 39, 3215.)

[36] Selective examples: (a) Nicewicz, D. A.; MacMillan, D. W. C. Science 2008, 322, 77.

(b) Prier, C. K.; Rankic, D. A.; MacMillan, D. W. C. Chem. Rev. 2013, 113, 5322.

(c) Yoon, T. P.; Ischay, M. A.; Du, J. Nat. Chem. 2010, 2, 527.

[37] Tan, H.; Li, H.; Ji, W.; Wang, L. Angew. Chem. Int. Ed. 2015, 54, 8374.

[38] Conner, E. S.; Crocker, K. E.; Fernando, R. G.; Fronczek, F. R.; Stanley, G. G.; Ragains, J. R. Org. Lett. 2013, 15, 5558.

[39] Shi, Q.; Li, P.; Zhang, Y.; Wang, L. Org. Chem. Front. 2017, 4, 1322 .

[40] Wei, W.; Cui, H.; Yang, D.; Yue, H.; He, C.; Zhang, Y.; Wang, H. Green Chem. 2017, 19, 5608.

[41] Yan, J.; Xu, J.; Zhou, Y.; Chen, J.; Song, Q. Org. Chem. Front.
2018, $5,1483$.

[42] Sahoo, H.; Mandal, A.; Dana, S.; Baidya, M. Adv. Synth. Catal. 2018, 360, 1099.

[43] Some examples: (a) Jin, D.-P.; Gao, P.; Chen, D.-Q.; Chen, S.; Wang, J.; Liu, X.-Y.; Liang, Y.-M. Org. Lett. 2016, 18, 3486. (b) Wen, J.; Wei, W.; Xue, S.; Yang, D.; Lou, Y.; Gao, C.; Wang, H. J. Org. Chem. 2015, 80, 4966.

[44] Ma, X-L.; Wang, Q.; Feng, X.-Y.; Mo, Z.-Y.; Pan, Y.-M.; Chen, Y.-Y.; Xin, M.; Xu, Y.-L. Green Chem. 2019, 21, 3547.

[45] Some examples: (a) Huang, M.-H.; Hao, W.-J.; Li, G.; Tu, S.-J.; Jiang, B. Chem. Commun. 2018, 54, 10791.

(b) Zhu, S.; Pathigoolla, A.; Lowe, G.; Walsh, D. A.; Cooper, M.; Lewis, W.; Lam, H. W. Chem.-Eur. J. 2017, 23, 17598.

(c) Kawaguchi, S.; Shirai, T.; Ohe, T.; Nomoto, A.; Sonoda, M.; Ogawa, A. J. Org. Chem. 2009, 74, 1751.

(d) Kobiki, Y.; Kawaguchi, S.; Ogawa, A. Tetrahedron Lett. 2013, 54,545

[46] Zhang, Q.; Yuan, P.; Kai, L.; Liu, K.; Ban, Y.; Wang, X.; Wu, L.; Liu, Q. Org. Lett. 2019, 21, 885.

[47] Some examples: (a) Ortgies, S.; Rieger, R.; Rode, K.; Koszinowski, K.; Kind, J.; Thiele, C. M.; Rehbein, J.; Breder, A. ACS Catal. 2017, 7, 7578 .

(b) Wilken, M.; Ortgies, S.; Breder, A.; Siewert, I. ACS Catal. 2018, 8, 10901 .

(c) Minakata, S.; Morino, Y.; Oderaotoshi, Y.; Komatsu, M. Org. Lett. 2006, 8, 3335.

(d) Nagaraju, K.; Rajesh, N.; Krishna, P. R. Synth. Commun. 2018, $48,1001$.

[48] Some examples: (a) Bin, D.; Wang, H.; Li, J.; Wang, H.; Yin, Z.; Kang, J.; He, B.; Li, Z. Electrochim. Acta 2014, 130, 170.

(b) Yin, Z.; Zheng, Y.; Wang, H.; Li, J.; Zhu, Q.; Wang, Y.; Ma, N.; Hu, G.; He, B.; Knop-Gericke, A.; Schlögl, R.; Ma, D. ACS Nano. 2017, 11, 12365 .

(c) Yan, M.; Kawamata, Y.; Baran, P. S. Chem. Rev. 2017, 117, 13230

(d) Zhang, Y.; Qi, Y.; Yin, Z.; Wang, H.; He, B.; Liang, X.; Li, J.; Li, Z. Green Chem. 2018, 20, 3944

(e) Sun, K.; Lei, J.; Liu, Y.-J.; Liu, B.; Chen, N. Adv. Synth. Catal. 2020, 362,1

[49] Guan, Z.; Wang, Y.; Wang, H.; Huang, Y.; Wang, S.; Tang, H.; Zhang, H.; Lei, A. Green Chem. 2019, 21, 4976.

[50] Some examples: (a) Wang, H.; Li, Y.; Lu, Q.; Yu, M.; Bai, X.; Wang, S.; Cong, H.; Zhang, H.; Lei, A. ACS Catal. 2019, 93, 1888. (b) Sun, L.; Yuan, Y.; Yao, M.; Wang, H.; Wang, D.; Gao, M.; Chen, Y.-H.; Lei, A. Org. Lett. 2019, 21, 1297.

(c) Zhang, Q.-B.; Yuan, P.-F.; Kai, L.-L.; Liu, K.; Ban, Y.-L.; Wang, X.-Y.; Wu, L.-Z.; Liu, Q. Org. Lett. 2019, 21, 885.

[51] Mallick, S.; Baidya, M.; Mahanty, K.; Maiti, D.; De Sarkar, S. Adv. Synth. Catal. 2020, 362, 1046.

[52] Hua, J.-W.; Fang, Z.; Xu, J.; Bian, M.-X.; Liu, C.-K.; He, W.; Zhu, N.; Guo, K. Green Chem. 2019, 21, 4706.

[53] Some examples: (a) Dakova, B.; Lamberts, L.; Evers, M. Electrochim. Acta 1994, 39, 2363.

(b) Kunai, A.; Harada, J.; Izumi, J.; Tachihara, H.; Sasaki, K. Electrochim. Acta 1983, 28, 1361.

(c) Torii, S.; Uneyama, K.; Ono, M.; Bannou, T. J. Am. Chem. Soc. 1981, 103, 4606.

[54] Hua, J.-W.; Fang, Z.; Bian, M.-X.; Ma, T.; Yang, M.; Xu, J.; Liu, C.-K.; He, W.; Zhu, N.; Yang, Z.; Guo, K. ChemSusChem 2020, 13, 2053.

[55] Some examples: (a) Torii, S.; Uneyama, K.; Ono, M.; Bannou, T. J. Am. Chem. Soc. 1981, 103, 4606.

(b) Dakova, B.; Lamberts, L.; Evers, M. Electrochim. Acta 1994, $39,2363$.

(c) Sun, K.; Wang, X.; Fu, F.; Zhang, C.; Chen, Y.; Liu, L. Green Chem. 2017, 19, 1490.

(d) Kim, Y. J.; Kim, D. Y. Org. Lett. 2019, 21, 1021

(e) Yang, X.-H.; Ouyang, X.-H.; Wei, W.-T.; Song, R.-J.; Li, J.-H. 
Adv. Synth. Catal. 2015, 357, 1161.

(f) Reddy, C. R.; Yarlagadda, S.; Ramesh, B.; Reddy, M. R.; Sridhar, B.; Reddy, B. V. S. Eur. J. Org. Chem. 2017, 2017, 2332.

[56] Guo, W.-S.; Wen, L.-R.; Li, M.; Wu, A.-G.; Liu, P.; Pan, C. Chin. J. Org. Chem. 2020, 40, 2855 (in Chinese) (郭维斯, 文丽荣, 李明, 武安国, 刘鹏, 潘超, 有机化学, 2020, 40, 2855.)

[57] Yue, D.; Larock, R. C. J. Org. Chem. 2002, 67, 1905

[58] Miao, M.; Huang, X. J. Org. Chem. 2009, 74, 5636.

[59] Gai, R.; Schumacher, R. F.; Back, D. F.; Zeni, G. Org. Lett. 2012, 14,6072 .

[60] Liu, J.; Li, P.; Chen, W.; Wang, L. Chem. Commun. 2012, 48, 10052.

[61] Some examples: (a) Wilhelm, E. A.; Jesse, C. R.; Prigol, M.; Alves, D.; Schumacher, R. F.; Nogueira, C. W. Cell Biol. Toxicol. 2010, 26,569 .

(b) Wilhelm, E. A.; Gai, B. M.; Souza, A. C. G.; Bortolatto, C. F.; Roehrs, J. A.; Nogueira, C. W. Mol. Cell. Biochem. 2012, 365, 175.

[62] Pistoia, R. P.; Roehrs, J. A.; Back, D. F.; Zeni, G. Adv. Synth. Catal. 2015, 357, 3655.

[63] Li, X.; He, P.; Zhou, H.; Dong, C. Org. Biomol. Chem. 2018, 16, 2150
[64] Wang, H.; Ying, J.; Lai, M.; Qi, X.; Peng, J.; Wu, X. Adv. Synth. Catal. 2018, 360, 1693.

[65] Fang, J.; Yan, X.; Zhou, L.; Wang, Y.; Liu, X. Adv. Synth. Catal. 2019, 361, 1985

[66] Some examples: (a) Sahoo, H.; Mandal, A.; Dana, S.; Baidya, M. Adv. Synth. Catal. 2018, 360, 1099.

(b) Sahoo, H.; Singh, S.; Baidya, M. Org. Lett. 2018, 20, 3678.

[67] Some examples: (a) Bravo, A.; Bjørsvik, H.-R.; Fontana, F.; Liguori, L.; Minisci, F. J. Org. Chem. 1997, 62, 3849.

(b) Shchepin, R.; Möller, M. N.; Kim, H.; Hatch, D. M.; Bartesaghi, S.; Kalyanaraman, B.; Radi, R.; Porter, N. A. J. Am. Chem. Soc. 2010, 132, 17490 .

(c) Hua, H.-L.; He, Y.-T.; Qiu, Y.-F.; Li, Y.-X.; Song, B.; Gao, P.; Song, X.-R.; Guo, D.-H.; Liu, X.-Y.; Liang, Y.-M. Chem.-Eur. J. 2015, 21, 1468.

(d) Smith, L. M.; Aitken, H. M.; Coote, M. L. Acc. Chem. Res. 2018, 51, 2006.

[68] Selected references: (a) Studer, A. Chem.-Eur. J. 2001, 7, 1159.

(b) Studer, A. Chem. Soc. Rev. 2004, 33, 267.

(c) Li, D.; Li, Y.; Yu, W. Synthesis 2017, 49, 4283

[69] Sahoo, H.; Grandhi, G. S.; Ramakrishna, I.; Baidya, M. Org. Biomol. Chem. 2019, 17, 10163.

(Zhao, C.) 